DOI: $10.24850 / j-$ tyca-2021-02-03

Artículos

\title{
Modelado físico y numérico de la interacción de ondas enfocadas con un dique vertical con banqueta baja
}

\section{Physical and numerical modeling of focused wave interactions with a low mound breakwater}

B. Rodrigo Covarrubias-Contreras ${ }^{1}$, ORCID: https://orcid.org/00000003-0297-176X

Alec Torres-Freyermuth², ORCID: https://orcid.org/0000-0003-02038734

José López-González³, ORCID: https://orcid.org/0000-0002-9977-3104

${ }^{1}$ Laboratorio de Ingeniería y Procesos Costeros, Instituto de Ingeniería, Universidad Nacional Autónoma de México, Sisal, Yucatán, México, BCovarrubiasC@iingen.unam.mx

²Laboratorio de Ingeniería y Procesos Costeros, Instituto de Ingeniería, Universidad Nacional Autónoma de México, Sisal, Yucatán, México, ATorresF@iingen.unam.mx

${ }^{3}$ Laboratorio de Ingeniería y Procesos Costeros, Instituto de Ingeniería, Universidad Nacional Autónoma de México, Sisal, Yucatán, México, JLopezGo@ii.unam.mx 


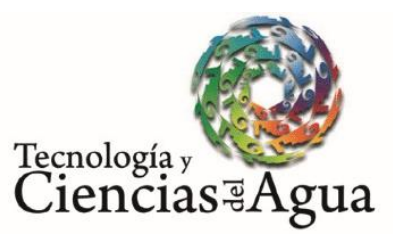

Autor para correspondencia: Alec Torres-Freyermuth, ATorresF@iingen.unam.mx

\section{Resumen}

Las ondas enfocadas, fenómeno que ocurre en el océano, son responsables del fallo de estructuras localizadas costa afuera. Existen pocos trabajos enfocados en su estudio en zonas cercanas a la costa. En el presente trabajo se analizó la estabilidad y funcionalidad de un dique vertical con banqueta baja en presencia de ondas enfocadas. Para ello, se realizaron ensayos en un canal de oleaje. Mediciones de superficie libre, presiones, subpresiones y rebase fueron utilizadas para validar un modelo numérico que resuelve las ecuaciones RANS. Las predicciones de rebase y presiones/subpresiones máximas del modelo numérico presentaron diferencias promedio con respecto a las mediciones de 10 y $8 \%$, respectivamente. De manera posterior, el modelo numérico se utilizó para evaluar el papel de la posición relativa de la estructura. Por último, se compararon los resultados del modelo físico con formulaciones semiempíricas, y se encontraron diferencias significativas en rebase $(\approx 100 \%)$, y en presiones y subpresiones ( $88 \%)$. Este estudio demuestra que el uso de modelos tipo RANS es una alternativa a las clásicas formulaciones semiempíricas, pues se comparan mejor con las mediciones durante este tipo de fenómenos. 


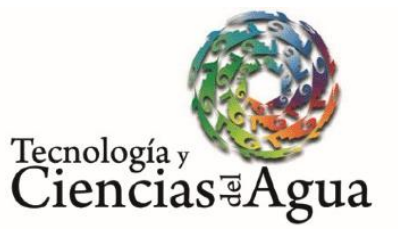

2021, Instituto Mexicano de Tecnología del Agua

Open Access bajo la licencia CC BY-NC-SA 4.0

(https://creativecommons.org/licenses/by-nc-sa/4.0/)

Palabras clave: ondas enfocadas, dique vertical con banqueta baja, funcionalidad, estabilidad estructural, ecuaciones RANS, rebase.

\section{Abstract}

Focused waves are a well-known phenomenon that occur in the ocean and can be responsible of offshore structure failure. However, less efforts have been devoted to improve its understanding in the nearshore. This work aims to investigate low-mound breakwater structure stability and functionality associated to transient wave groups. Laboratory experiments were conducted in a wave flume. Free-surface elevation, pressures, and overtopping measurements were employed to validate a numerical model based on the RANS equations. The numerical model predicts the pressures and overtopping with an average relative error of 10 and $8 \%$, respectively. Subsequently, the numerical model was employed to evaluate the role of the relative structure position with respect to the theoretical focused point. Finally, the physical model results are compared with semi-empirical formulations, finding significant differences for both wave overtopping $(\approx 100 \%)$ and pressure/sub-pressure (88 \%) estimates. This work demonstrates that RANS models are an alternative to the classic formulations that failed to predict stability and functionality during these phenomena.

Keywords: Focus wave, rouge waves, vertical breakwater, structure functionality, structural stability, RANS equations, overtopping. 


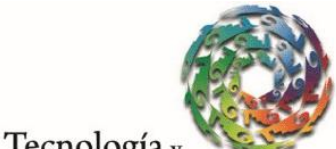 \\ Tecnología y \\ Ciencias Agua}

Recibido: $11 / 03 / 2020$

Aceptado: $24 / 06 / 2020$
2021, Instituto Mexicano de Tecnología del Agua

Open Access bajo la licencia CC BY-NC-SA 4.0

(https://creativecommons.org/licenses/by-nc-sa/4.0/)

\section{Introducción}

La zona costera es altamente vulnerable ante eventos extremos y el incremento del nivel medio del mar asociado con el cambio climático (Gornitz, 1991). Las estructuras costeras brindan protección a la infraestructura y permiten el desarrollo de diferentes actividades económicas en dicha área (desarrollos turísticos, zonas industriales, y operaciones portuarias). El régimen de oleaje medio y extremal es fundamental para el diseño de cualquier obra de protección costera (ROM 0.0). El diseño debe garantizar la funcionalidad y estabilidad ante la ocurrencia de eventos extremos asociados con cierto periodo de retorno (Goda, 2004).

El oleaje es un fenómeno aleatorio que se puede caracterizar con parámetros en el dominio del tiempo y de la frecuencia (p. ej., $H_{s}, T_{p}$ ). Conociendo los parámetros del oleaje y suponiendo un espectro teórico se pueden generar un número infinito de series de tiempo de superficie 


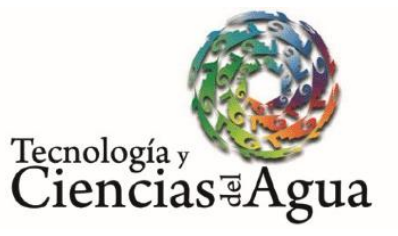

2021, Instituto Mexicano de Tecnología del Agua

Open Access bajo la licencia CC BY-NC-SA 4.0

(https://creativecommons.org/licenses/by-nc-sa/4.0/)

libre (Palemón-Arcos, Torres-Freyermuth, Pedrozo-Acuña, \& Salles, 2015), ya que los parámetros espectrales no proveen de la información (fase) necesaria para la reconstrucción de la serie original del tren de olas. Esta información puede ser relevante, pues en la realidad la interacción entre las diferentes componentes afecta la evolución espacio-temporal del tren de olas. Las componentes pueden interactuar de manera positiva o negativa. Un ejemplo de dicha interacción es la generación de ondas enfocadas. Este fenómeno sucede cuando las componentes del grupo de ondas transitorias entran en fase en un lugar en el espacio. Esto puede dar como resultado oleaje extremo conocido como "rogue waves" (Stagonas, Buldakov, \& Simons, 2018). Este tipo de ondas en los registros se caracterizan por ser una onda solitaria, notablemente asimétrica en su horizontalidad, que presenta una ola extrema (Van-den-Boomgaard, 2003). Esta ola extrema es comúnmente de 1.8 veces la altura de ola significante (Nikolkina \& Didenkulova, 2011).

La mayoría de los estudios con ondas enfocadas se han desarrollado para aguas profundas e intermedias, utilizando modelos físicos (Ryu \& Chang, 2008) o numéricos (Amarachaharam, 2016; Palemón-Arcos, Torres-Freyermuth, Chang, Pastrana-Maldonado, \& Salles, 2014; Li, Zhao, Ye, Lin, \& Chen, 2018). Esto se debe a que dicho fenómeno ha sido responsable del daño a infraestructura asociada con la industria de extracción de hidrocarburos y embarcaciones. Sin embargo, pocos trabajos se han enfocado en conocer las implicaciones de las ondas enfocadas en zonas cercanas a la costa (Whittaker et al., 2017; Whittaker, Fitzgerald, Raby, Taylor, \& Borthwick, 2018). Estudios recientes (Whittaker et al., 2017; Whittaker et al., 2018) han demostrado la importancia de las ondas enfocadas en el estudio de playas y muros 


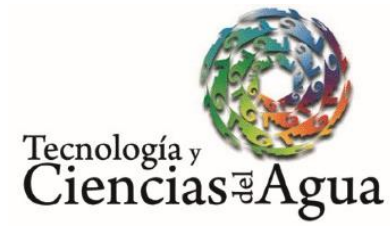

2021, Instituto Mexicano de Tecnología del Agua

Open Access bajo la licencia CC BY-NC-SA 4.0

(https://creativecommons.org/licenses/by-nc-sa/4.0/)

inclinados utilizando un modelo tipo Boussinesq. Sin embargo, la capacidad de modelos numéricos más avanzados (p. ej., RANS) y simplificados (p. ej., modelos semianalíticos/empíricos) para cuantificar la funcionalidad y estabilidad de diferentes tipologías ante este tipo de eventos requiere mayor atención.

Una de las herramientas más usadas para el diseño de estructuras costeras es el modelado físico (Jiménez, 2010; Guanche, 2007), y el uso de formulaciones semiempíricas y paramétricas (Goda, 1974; Franco \& Franco, 1999). En años recientes ha cobrado importancia el uso de modelos numéricos basados en las ecuaciones RANS (Lara, García, \& Losada, 2006; Losada, Lara, Guanche, \& González-Ondina, 2008; Guanche, Losada, \& Lara, 2009; Higuera, Lara, \& Losada, 2014; PalemónArcos et al., 2015), que resuelven la transformación no-lineal del oleaje, incluyendo la rotura. En el presente trabajo se analizará la estabilidad y funcionalidad de un dique vertical de banqueta baja al interactuar con ondas enfocadas a través del modelado físico y numérico. Asimismo, las mediciones serán comparadas formulaciones semianalíticas/empíricas existentes utilizadas para el estudio de estabilidad y rebase en estas estructuras costeras.

\section{Métodos}




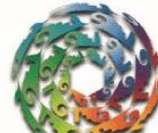

Ciencias ฐ̊tgua
2021, Instituto Mexicano de Tecnología del Agua

Open Access bajo la licencia CC BY-NC-SA 4.0

(https://creativecommons.org/licenses/by-nc-sa/4.0/)

El presente trabajo contempla un estudio integral que incluye: (a) el desarrollo de ensayos en un modelo físico; (b) la implementación, validación, y aplicación de un modelo numérico; y (c) la evaluación de formulaciones semiempíricas para estimar las presiones/subpresiones y el rebase en un dique vertical. La metodología utilizada se ilustra en la Figura 1 y se detalla a continuación.

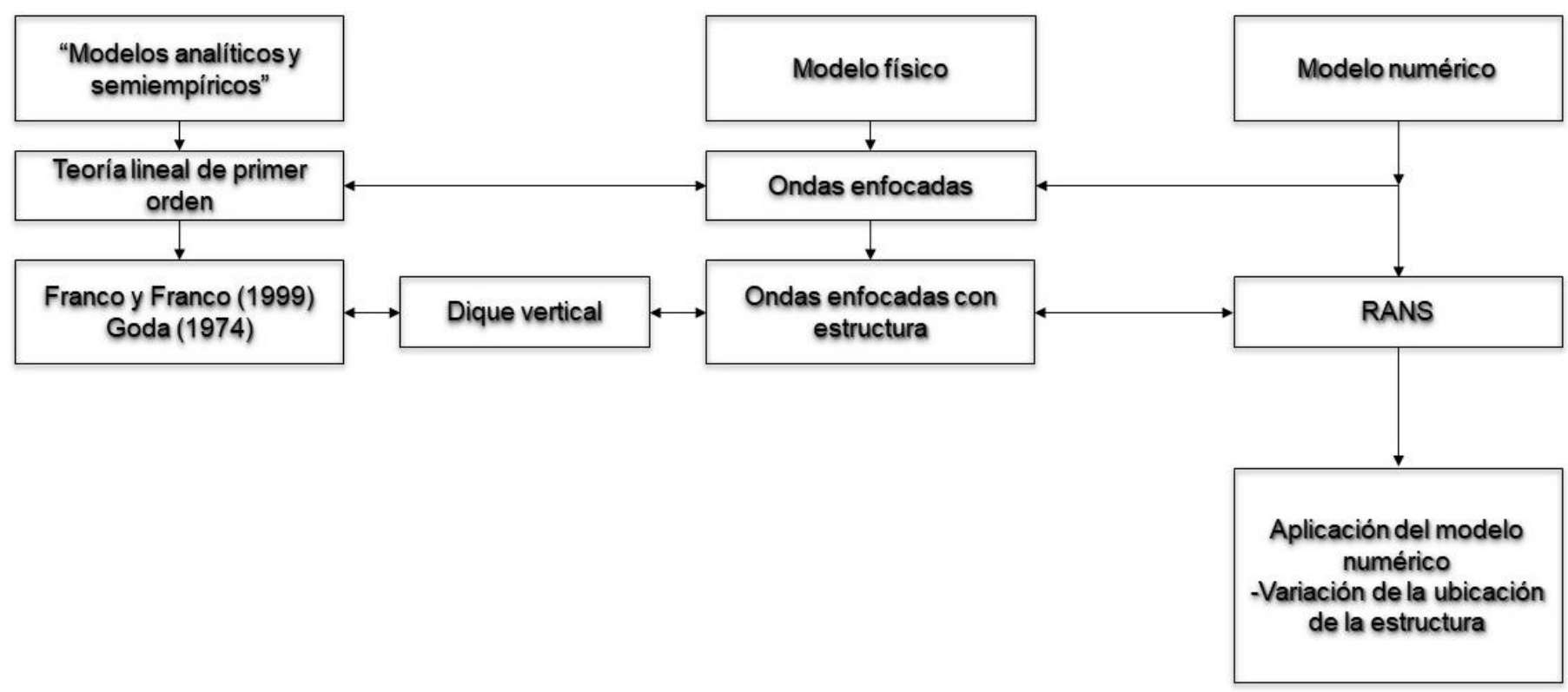

Figura 1. Diagrama de flujo de la metodología para modelar física, numéricamente, y a través de modelos analíticos y semiempíricos la interacción de ondas enfocadas con un dique vertical. 


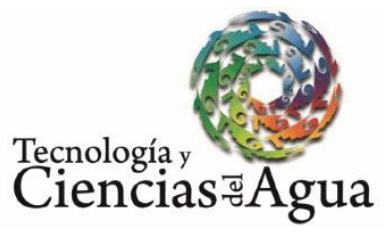

\section{Generación de ondas enfocadas}

La metodología de generación de ondas enfocadas en este trabajo se basa en la teoría de onda lineal propuesta por Longuet-Higgins (1974). Este método de generación de ondas enfocadas, basado en la teoría lineal, ha sido utilizado en estudios previos (Longuet-Higgins, 1974; Rapp \& Melville, 1990; Baldock, Swan, \& Taylor, 1996; Baldock, 2006; entre otros). El método considera un espectro de oleaje que se subdivide en $N$ componentes que contienen la misma energía en el dominio de la frecuencia. El objetivo es generar un rango específico de componentes de ondas que entren en fase (enfocamiento) en una ubicación específica y tiempo determinado, generando una ola de mayor altura, producto de la superposición de componentes. La elevación de la superficie libre del agua se puede expresar como la suma de componentes, dada por:

$\eta(x, t)=\sum_{i=1}^{N} a_{i} \cos \left(k_{i} x-\omega_{i} t-\phi_{i}\right)$

donde $a_{i}, k_{i}, \omega_{i}=2 \pi f_{i}$, y $\phi_{i}$ son la amplitud, el número de onda, la frecuencia angular, y la fase de la i-ésima componente; $N$ es el número de componentes y $t$ es el tiempo. Donde $k_{i}$ y $\omega_{i}$ están relacionados por la ecuación de la dispersión $\omega_{i}{ }^{2}=g k_{i} \tanh \left(k_{i} h\right)$, en donde $g$ y $h$ son la aceleración de la gravedad y la profundidad del agua, respectivamente. La fase de cada uno de los componentes se calcula estableciendo: 
$\cos \left(k_{i} x-\omega_{i} t-\phi_{i}\right)=1$

en donde $x=x_{f}$ será el punto de enfoque y $t=t_{f}$ el tiempo de enfocamiento, quedando la Ecuación (2) como:

$\phi_{i}=k_{i} x_{f}-\omega_{i} t_{f}$

Sustituyendo (3) en (1), obtenemos que el desplazamiento de la superficie libre está dado por:

$\eta(x, t)=\sum_{i=1}^{N} a_{i} \cos \left(k_{i}\left(x-x_{f}\right)-\omega_{i}\left(t-t_{f}\right)\right)$

donde la posición media de la pala generadora de olas se define en $x=0$ (ver Figura 2c) y el desplazamiento deseado en la pala generadora de olas está dado por:

$\eta\left(0, t^{\prime}\right)=\sum_{i=1}^{N} a_{i} \cos \left(-k_{i} x_{f}-\omega_{i} t^{\prime}\right)$

donde $t^{\prime}=t-t_{f}$ y el efecto de cambiar $t_{f}$ es retrasar o avanzar toda la señal sin cambiar el punto de enfoque del grupo de ondas teórico $x_{f}$. 

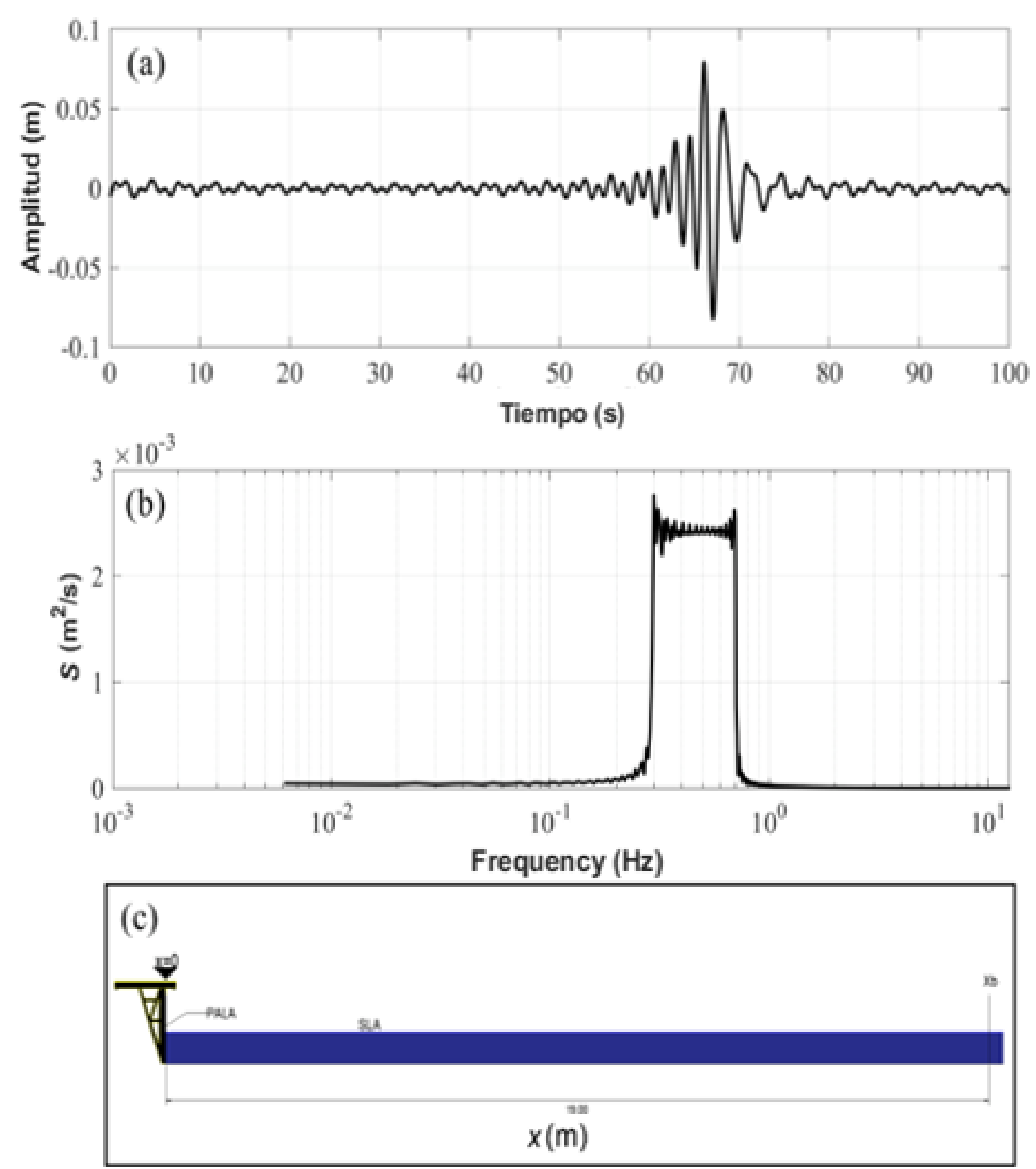

Figura 2. (a) Grupo de ondas enfocadas en aguas intermedias utilizando 32 componentes con una $f_{c}=1 \mathrm{~Hz}, f_{1}=0.3 \mathrm{~Hz}, f_{2}=0.7 \mathrm{~Hz}$ y $A=0.06 \mathrm{~m}$, tomando en cuenta un punto de enfoque en $x=19 \mathrm{~m}$; (b) espectro tipo "sombrero"; (c) geometría del canal, señalando el sistema de referencia utilizado con origen $(x=0 \mathrm{~m})$ en la posición de la pala. 


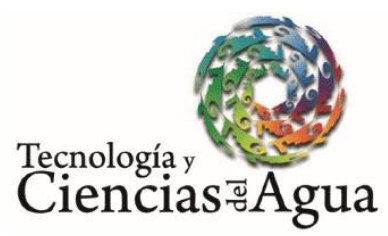

2021, Instituto Mexicano de Tecnología del Agua

Open Access bajo la licencia CC BY-NC-SA 4.0

(https://creativecommons.org/licenses/by-nc-sa/4.0/)

Considerando las características del canal de oleaje se generó un grupo de ondas conformado por 32 componentes. Estudios previos (Rapp \& Melville, 1990; Baldock et al., 1996; Baldock, 2006) demostraron una buena representación de este tipo de oleaje utilizando alrededor de 30 componentes. La frecuencia central $f_{c}=0.5 \mathrm{~Hz}$, inferior $f_{1}=0.3 \mathrm{~Hz}$ y superior $f_{2}=0.7 \mathrm{~Hz}$ se usaron para generar la serie temporal de superficie libre para este estudio (Figura 2a). El tren de ondas generado dio como resultado un espectro tipo "sombrero" (Figura 2b), característico en este tipo de grupo de ondas. El punto de enfoque teórico se localizó en $x=$ $19 \mathrm{~m}$ (Figura 2c) para un tiempo de cien segundos.

\section{Modelo físico}

Los ensayos de laboratorio se llevaron a cabo en el canal de oleaje del Laboratorio de Ingeniería y Procesos Costeros (LIPC) de la Unidad Académica Sisal del Instituto de Ingeniería (II) de la Universidad Nacional Autónoma de México (UNAM). El canal de oleaje tiene $40 \mathrm{~m}$ de longitud, $0.8 \mathrm{~m}$ de ancho y $1.27 \mathrm{~m}$ de alto (Figura 3), y está equipado con un generador de oleaje unidireccional (GOU) del fabricante VTI, el cual consiste de una pala tipo pistón, con una potencia de $7.5 \mathrm{KW}$ y una carrera de la pala de $1.2 \mathrm{~m}$. El sistema de generación cuenta con generación de 
segundo orden y absorción activa. En este trabajo se utilizó una serie temporal de superficie libre (Figura 2a) para la generación del oleaje.

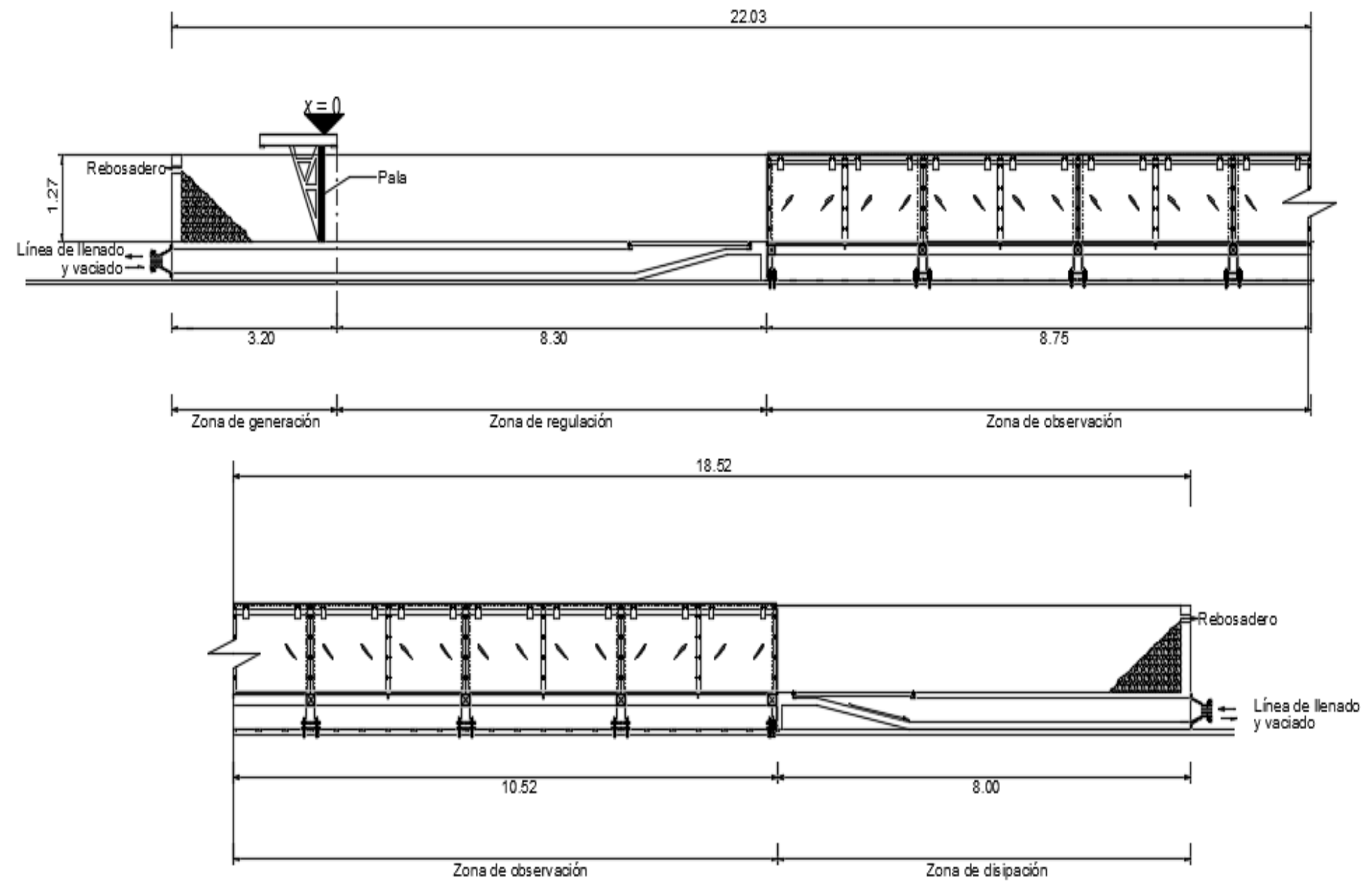

Figura 3. Esquema de la geometría del canal. 


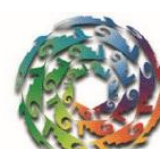

Ciencias $₫$ Agua
2021, Instituto Mexicano de Tecnología del Agua

Open Access bajo la licencia CC BY-NC-SA 4.0

(https://creativecommons.org/licenses/by-nc-sa/4.0/)

El diseño de la tipología de la estructura costera modelada físicamente en el canal del LIPC del II-UNAM corresponde a un dique vertical con banqueta baja (Low Mound Breakwater) a escala 1:20 (Figura 4). El dique y el vertedero para medir el rebase se construyeron con tablas de madera de 3/8". El vertedero se forró por dentro con láminas de acrílico y se selló con silicón para evitar fugas. Para la banqueta del dique se usó roca caliza y una pendiente de 1:2 (Figura 4).

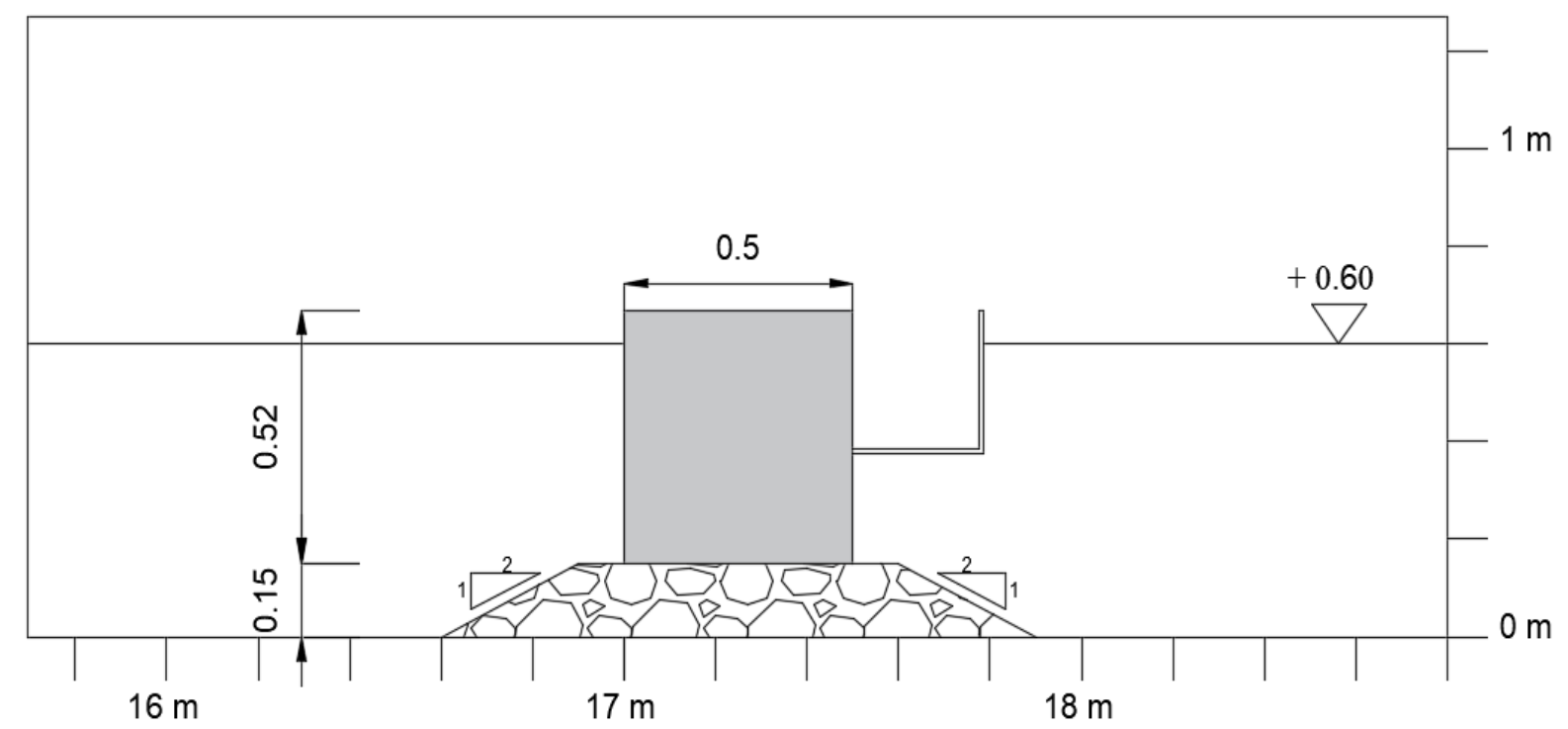

Figura 4. Sección transversal del dique vertical. 


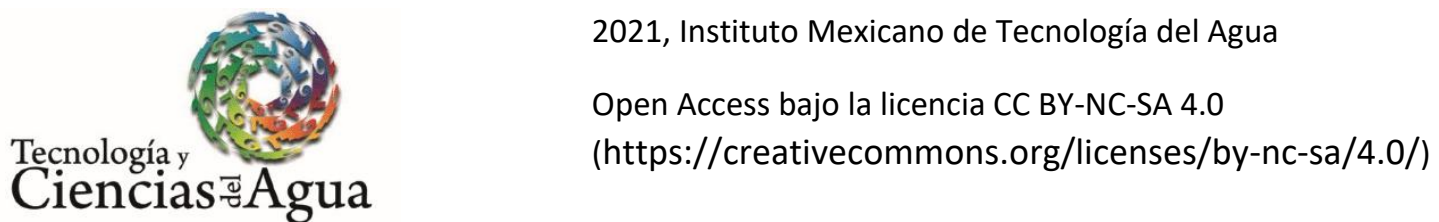

En el modelo físico se realizaron tres ensayos a escala (1:20): (a) el primero consistió en la generación de un grupo de ondas enfocadas y propagadas a una profundidad constante de $0.60 \mathrm{~m}$ sin la presencia de la estructura; (b) el segundo fue con las mismas condiciones que en (a), pero con la estructura ubicada en el punto de enfoque observado $x=17$ $\mathrm{m}$, que presenta una diferencia de $2 \mathrm{~m}$ con respecto al punto de enfoque teórico; c) el tercero consistió en la interacción del grupo de ondas enfocadas con el mismo oleaje y la misma tipología, pero con un nivel del agua en reposo de $0.50 \mathrm{~m}$. Para los ensayos (b) y (c) se hicieron 15 repeticiones, a fin de calcular los promedios de ensamble. La altura de ola significante $\left(H_{s}\right)$, periodo pico $\left(T_{p}\right)$, y nivel del agua en reposo $(h)$ para cada uno de los casos simulados se especifica en la Tabla 1.

Tabla 1. Ensayos realizados en el canal de olas del LIPC de la UNAM.

\begin{tabular}{|c|c|c|c|c|c|c|}
\hline Ensayo & $\begin{array}{c}h \\
(m)\end{array}$ & Tipo de oleaje & $\begin{array}{c}H_{s} \\
(\mathrm{~m})\end{array}$ & $\begin{array}{l}T_{p} \\
(s)\end{array}$ & $\begin{array}{c}\text { Estructura } \\
\text { (m) }\end{array}$ & Repeticiones \\
\hline 1 & 0.60 & Ondas enfocadas & 0.04 & 2.00 & $\begin{array}{c}\text { Sin } \\
\text { estructura }\end{array}$ & 4 \\
\hline 2 & 0.60 & Ondas enfocadas & 0.04 & 2.00 & 17.00 & 15 \\
\hline 3 & 0.50 & Ondas enfocadas & 0.04 & 2.00 & 17.00 & 15 \\
\hline
\end{tabular}

*Donde $h=$ profundidad en reposo; $H_{s}=$ altura significante y $T_{p}=$ periodo de pico. 


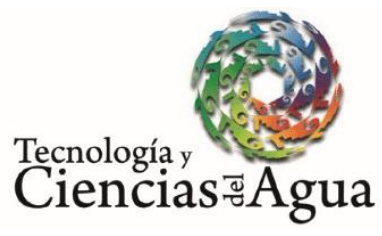

\section{Adquisición y análisis de los datos}

Para medir la transformación del oleaje se colocaron 21 sensores de nivel tipo resistivo fabricados por VTI, utilizando una frecuencia de muestreo de $100 \mathrm{~Hz}$ (Figura 5). Asimismo, se realizaron mediciones de presión con 10 sensores colocados alrededor de la estructura (Figura 6) para la medición de presiones y subpresiones (cinco en el muro vertical y cinco en la base). Los sensores utilizados fueron marca Keller America, con una precisión de $\pm 0.25 \% \circ \pm 0.1 \%$ TEB; un intervalo de presión de 0 a 39 225.53 Pascales, y un intervalo de temperatura de -10 a $80^{\circ} \mathrm{C}$. En los ensayos 2 y 3 se utilizó una frecuencia de muestreo para la presión de 19 $\mathrm{Hz}$. Las frecuencias de muestreo utilizadas para cada variable estuvieron en función de la frecuencia de muestreo de los sensores de medición. Por otro lado, para medir el nivel de agua en la cubeta de rebase y estimar el volumen de rebase y gasto, se empleó un sensor resistivo, acústico y de presión.

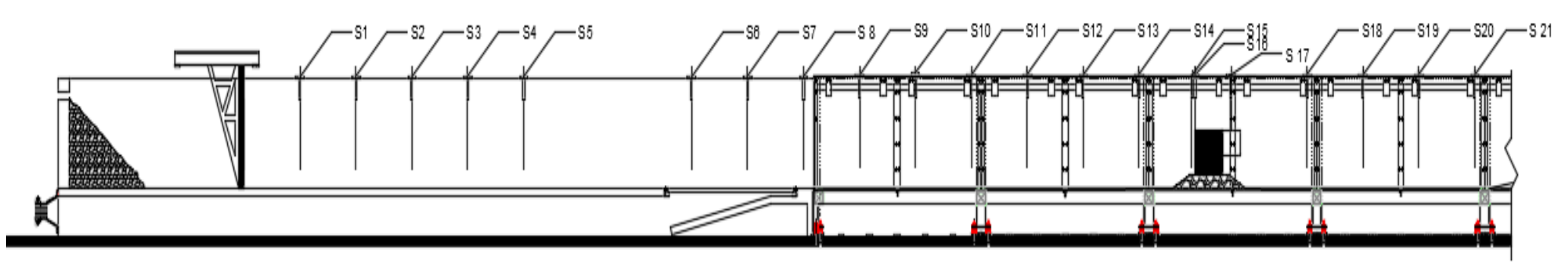




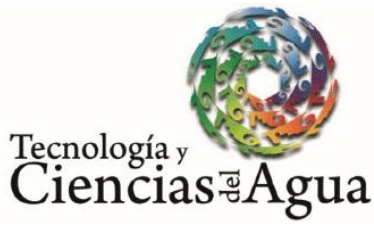

2021, Instituto Mexicano de Tecnología del Agua

Open Access bajo la licencia CC BY-NC-SA 4.0

(https://creativecommons.org/licenses/by-nc-sa/4.0/)

Figura 5. Plano de ubicación de los sensores de nivel en los ensayos de laboratorio. El ensayo 1 tiene el mismo arreglo, pero sin la estructura y con el sensor S16 ubicado a $0.5 \mathrm{~m}$ del S15.

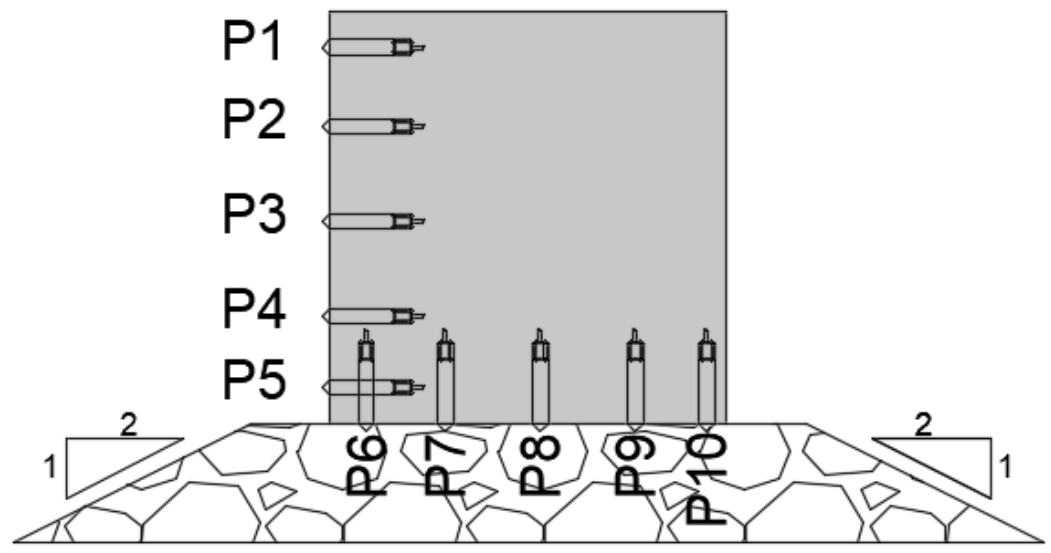

Figura 6. Plano de ubicación de los sensores de presión.

Se realizaron las curvas de calibración para todos los sensores resistivos antes de cada uno de los ensayos, a fin de convertir las mediciones de voltaje a metros. Para ello, se generaron curvas de calibración a partir de la medición de diferentes niveles conocidos y su posterior ajuste a través de una regresión lineal, obteniendo un coeficiente de correlación promedio de 0.99. Las series temporales de superficie libre se analizaron a través del método de cruce ascendentes por ceros para encontrar las alturas de ola en el tren de oleaje, y a partir de ellas determinar en cada sensor la altura de ola máxima y la altura de ola significante. En el caso de los sensores de presión no se requiere 


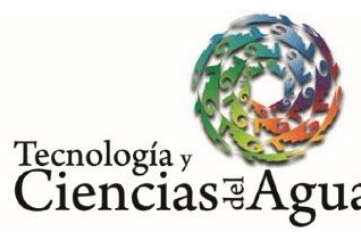

2021, Instituto Mexicano de Tecnología del Agua

Open Access bajo la licencia CC BY-NC-SA 4.0

(https://creativecommons.org/licenses/by-nc-sa/4.0/)

ninguna calibración y, por lo tanto, se obtuvieron los valores promedio y máximos a partir del registro. El rebase se estimó a partir del nivel de agua en la cubeta por medio de un sensor resistivo, acústico y de presión.

\section{Modelo numérico}

Para el modelado numérico se utilizó el modelo Cornell Breaking Wave and Structures (COBRAS) (Lin \& Liu, 1998). Este modelo fue desarrollado originalmente por la Universidad de Cornell a partir del modelo de la NASA Ilamado RIPPLE. En estudios posteriores (Hsu, Sakakiyama, \& Liu, 2002; Lara et al., 2006; Losada et al., 2008), el modelo COBRAS fue adaptado para el estudio de la interacción de flujo-estructura para cualquier tipología permeable o impermeable.

El modelo bidimensional (2DV) COBRAS resuelve las ecuaciones de Reynolds y Navier Stokes promediadas en el volumen (VARANS); utiliza un modelo de turbulencia $k-\varepsilon$ algebraico no lineal y describe la superficie libre sin restricciones geométricas por medio de la técnica VOF (Volume of Fluid), por lo cual se pueden representar todos los procesos de trasformación del oleaje, incluida la reflexión, la rotura, y el rebase. Este modelo permite obtener el campo de presiones no-hidrostático, reduciendo el número de limitaciones, a diferencia de los modelos de 


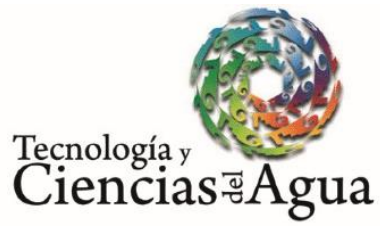

2021, Instituto Mexicano de Tecnología del Agua

Open Access bajo la licencia CC BY-NC-SA 4.0

(https://creativecommons.org/licenses/by-nc-sa/4.0/)

ecuaciones no-lineales de aguas someras (Kobayashi \& Wurjanto, 1992) o tipo Boussinesq (Chen, Kirby, Dalrymple, Shi, \& Thornton, 2003), debido a que no asume ninguna teoría de ondas.

En este trabajo, el modelo numérico se validó con los datos de superficie libre y presión obtenidos en el canal de oleaje. Asimismo, el modelo se usó para estudiar el papel de la ubicación relativa de la estructura, con relación al punto teórico de enfocamiento del grupo de ondas, en la estabilidad y funcionalidad de la tipología estudiada. Por lo tanto, el dominio de cómputo y las condiciones de contorno se implementaron para reproducir las condiciones del canal de oleaje.

El modelo numérico fue forzado con las mediciones de superficie libre medidas en el sensor más cercano a la pala de generación del canal de oleaje (S1 en Figura 5). Para derivar la condición de flujo, la serie temporal es descompuesta en sus componentes de Fourier, con el objetivo de derivar el perfil de velocidades de cada una de las componentes utilizando teoría lineal (Torres-Freyermuth, Losada, \& Lara, 2007). Por lo tanto, la serie temporal del perfil de velocidades se construye por superposición lineal. El modelo numérico utiliza absorción activa para evitar la re-reflexión del oleaje en el contorno.

Se modelaron seis casos (ver Tabla 2), donde el primero corresponde a un grupo de ondas enfocadas sin estructura, con el fin de evaluar el método de generación en el modelo numérico y su capacidad para predecir el punto de enfocamiento en el modelo físico. El segundo ensayo estuvo enfocado al estudio de la interacción del grupo de ondas enfocadas con la tipología propuesta; este caso permitió validar el modelo numérico con las mediciones de superficie libre, presiones, y rebase 


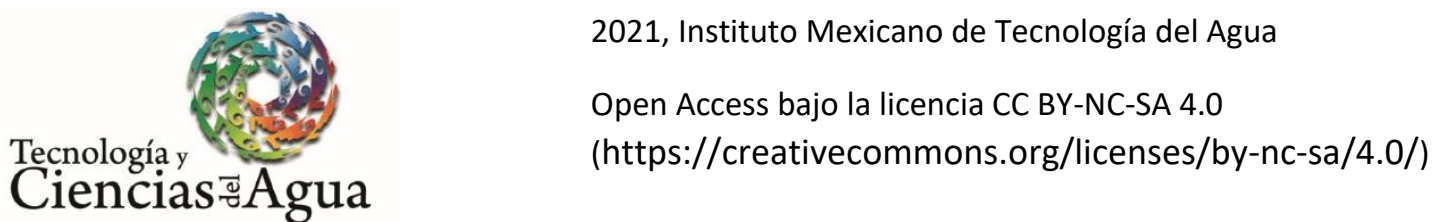

obtenidas en el laboratorio. Los casos 3 a 6 mantuvieron las mismas condiciones de forzamiento que en el caso 2, pero variando la ubicación de la estructura. Esto permitió evaluar la estabilidad y funcionalidad del dique, considerando la variación de su posición con respecto al punto de enfocamiento teórico en ausencia de la estructura (ver Tabla 2). Para todos los casos se utilizó un dominio computacional de $30 \mathrm{~m}$ de largo y $1.3 \mathrm{~m}$ de alto. La malla utilizada fue uniforme horizontal y vertical, con resolución de $\Delta x=0.02 \mathrm{~m}$ y $\Delta y=0.01 \mathrm{~m}$. El número total de celdas es de 1500 en $x$ y 130 en $y$. El tamaño de la malla se seleccionó a través de un análisis de sensibilidad y considerando el tiempo de cómputo.

Tabla 2. Casos modelados en COBRAS.

\begin{tabular}{|c|c|c|c|c|c|c|}
\hline Caso & $\boldsymbol{h}$ & Tipo de oleaje & $\boldsymbol{H}_{\boldsymbol{s}}$ & $\boldsymbol{T}_{\boldsymbol{p}}$ & Estructura & Repeticiones \\
\hline $\mathbf{1}$ & $\mathbf{( m )}$ & & $\mathbf{( m )}$ & $\mathbf{( s )}$ & $\mathbf{( m )}$ & 1 \\
\hline $\mathbf{2}$ & 0.60 & Ondas enfocadas & 0.04 & 2.00 & $\begin{array}{c}\text { Sin } \\
\text { estructura }\end{array}$ & \\
\hline $\mathbf{3}$ & 0.60 & Ondas enfocadas & 0.04 & 2.00 & 7.00 & 1 \\
\hline $\mathbf{4}$ & 0.60 & Ondas enfocadas & 0.04 & 2.00 & 14.00 & 1 \\
\hline $\mathbf{5}$ & 0.60 & Ondas enfocadas & 0.04 & 2.00 & 20.00 & 1 \\
\hline $\mathbf{6}$ & 0.60 & Ondas enfocadas & 0.04 & 2.00 & 27.00 & 1 \\
\hline
\end{tabular}




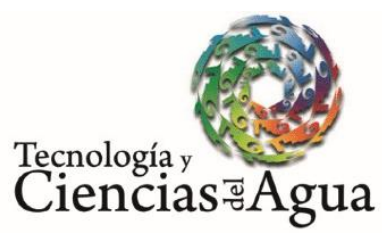

2021, Instituto Mexicano de Tecnología del Agua

Open Access bajo la licencia CC BY-NC-SA 4.0

(https://creativecommons.org/licenses/by-nc-sa/4.0/)

Formulaciones semiempíricas

En este trabajo se utilizaron formulaciones semiempíricas existentes para el estudio de estabilidad y funcionalidad de estructuras con la tipología de estudio. El objetivo fue comparar su desempeño con respecto a las mediciones y el modelo numérico durante la presencia de ondas enfocadas.

Para el estudio de estabilidad, ante oleaje pulsante, se utilizó el modelo de Goda (1974). Este modelo utiliza para el cálculo de la sobreelevación y las presiones las siguientes expresiones:

$H=1.8 * H_{1 / 3}$

$\eta^{*}=0.75(1+\cos \beta) H$

$p_{1}=0.5(1+\cos \beta)\left(\alpha_{1}+\alpha_{2} \cos ^{2} \beta\right) \rho_{w} g H$

$p_{2}=\frac{p_{1}}{\cosh \left(\frac{2 \pi h}{L}\right)}$

$p_{3}=\alpha_{3} p_{1}$ 


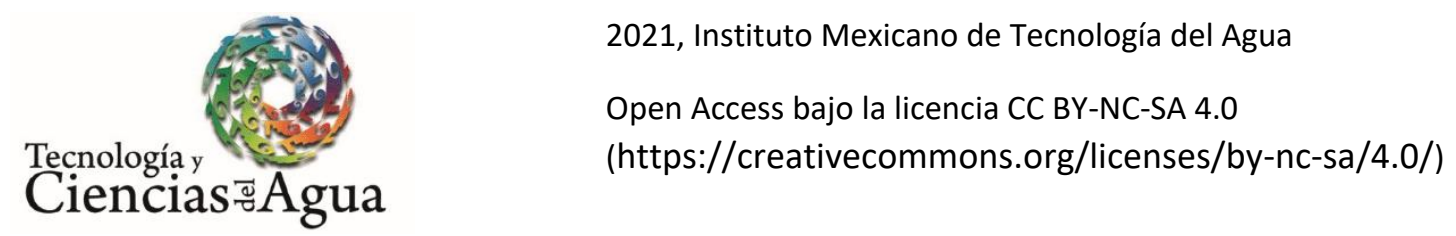

$p_{u}=0.5(1+\cos \beta) \alpha_{1} \alpha_{3} \gamma_{w} H$

Los valores de los distintos coeficientes $\alpha_{i}$ están dados por:

$\alpha_{1}=0.6+0.5\left[\frac{4 \pi \frac{h}{L}}{\operatorname{senh}\left(4 \pi \frac{h}{L}\right)}\right]^{2}$
$\alpha_{2}=\min \left\{\left[\frac{h_{b}-d}{3 h_{b}}\left(\frac{H}{d}\right)^{2}, \frac{2 d}{H}\right]\right\}$

Donde:

$h_{b}=h+5 H_{1 / 3} \tan \theta$

$\alpha_{3}=1-\frac{h^{\prime}}{h}\left[1-\frac{1}{\cos \left(2 \pi \frac{h}{L}\right)}\right]^{2}$

Las variables utilizadas en el modelo se muestran en la Figura 7 y los parámetros empleados corresponden a las características de los ensayos hechos en el canal de oleaje (Tabla 3). 


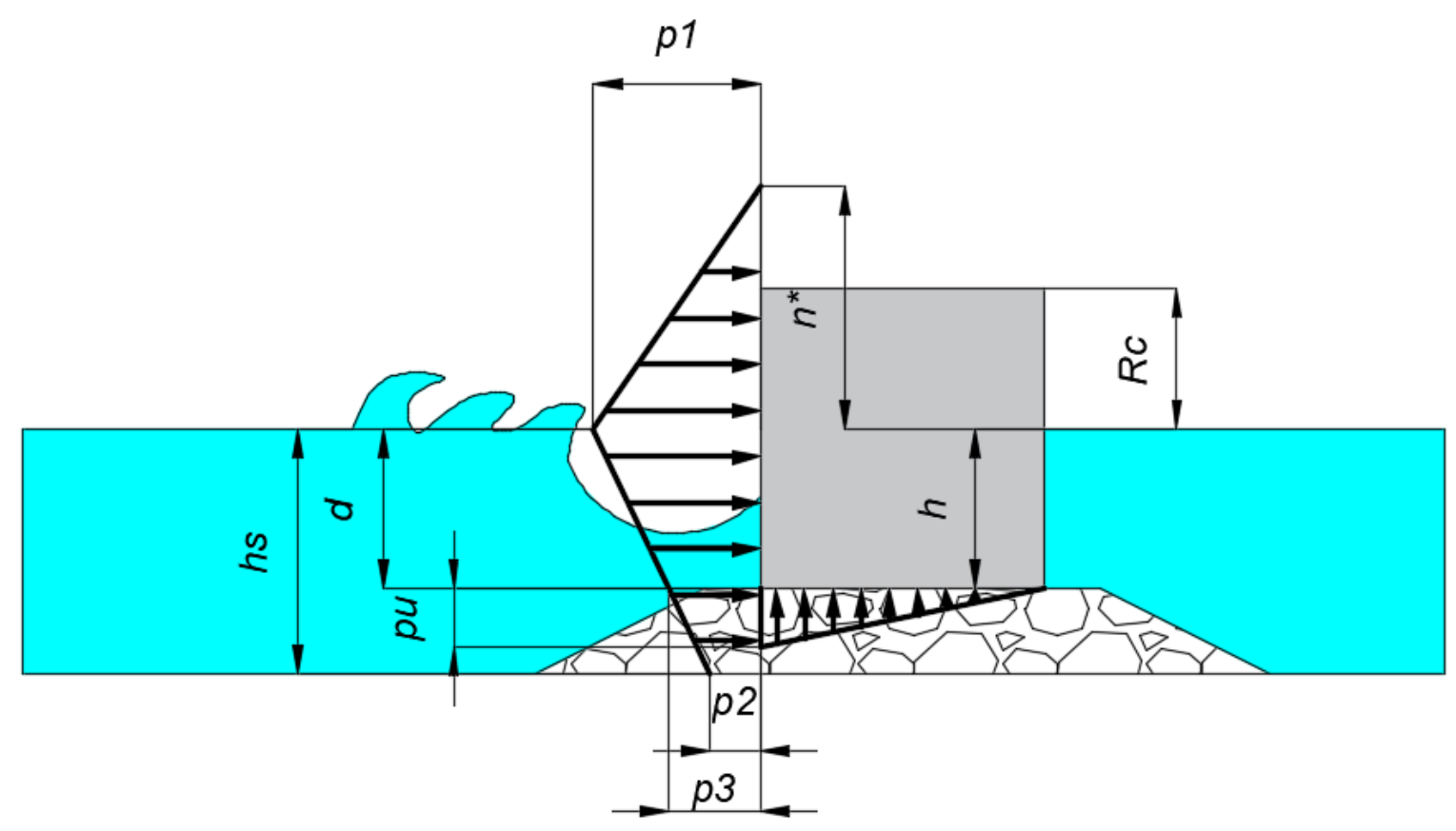

Figura 7. Diagrama de presiones de Goda (1974).

Tabla 3. Parámetros utilizados en el método de Goda (1974).

\begin{tabular}{|c|c|c|c|c|c|c|c|c|}
\hline Ensayo & $\begin{array}{l}\text { hs } \\
\text { (m) }\end{array}$ & Tipo de oleaje & $\begin{array}{c}H_{s} \\
(\mathrm{~m})\end{array}$ & $\begin{array}{c}H \\
(\mathrm{~m})\end{array}$ & $\begin{array}{c}T_{p} \\
\text { (s) }\end{array}$ & $\begin{array}{l}\text { hc } \\
\text { (m) }\end{array}$ & $\begin{array}{c}h \\
(\mathrm{~m})\end{array}$ & $\boldsymbol{\beta}$ \\
\hline 2 & 0.60 & $\begin{array}{c}\text { Ondas } \\
\text { enfocadas }\end{array}$ & 0.04 & 0.80 & 2.00 & 0.07 & 0.45 & 0 \\
\hline 3 & 0.50 & $\begin{array}{c}\text { Ondas } \\
\text { enfocadas }\end{array}$ & 0.04 & 0.80 & 2.00 & 0.17 & 0.45 & 0 \\
\hline
\end{tabular}

*Donde $S L=$ superficie libre; $H_{s}=$ altura significante; $T_{p}=$ periodo pico; $h s=$ calado a pie de estructura; $h=$ calado, y $\beta=$ ángulo de incidencia del oleaje $\left({ }^{\circ}\right)$. 
Para el cálculo de rebase en un dique vertical se utilizó la formulación de Franco y Franco (1999) dada por:

$Q=\frac{q}{\sqrt{g H_{s}^{3}}}=0.082 \exp \left(-3.0 \frac{R c}{H_{s}} \frac{1}{\gamma_{\beta} \gamma_{s}}\right)$

Donde:

$Q=$ caudal medio de rebase adimensional.

$q=$ caudal medio de rebase $\left(\mathrm{m}^{3} / \mathrm{s} / \mathrm{m}\right)$.

$g=$ aceleración de la gravedad $\left(\mathrm{m}^{2} / \mathrm{s}\right)$.

$R c=$ francobordo $(\mathrm{m})$.

$H s=$ altura de ola significante $(\mathrm{m})$.

$\gamma_{\beta}=$ coeficiente adimensional función del ángulo de incidencia del oleaje.

$\gamma_{\mathrm{s}}=$ coeficiente adimensional de la geometría frontal del paramento.

Para el cálculo de rebase con la ecuación de Franco y Franco (1999) se utilizaron los siguientes parámetros, que se muestran en la Tabla 4 de los ensayos 2 y 3. 
Tabla 4. Parámetros utilizados en el método de Franco y Franco (1999).

\begin{tabular}{|c|c|c|c|c|c|c|c|}
\hline Ensayo & $\begin{array}{l}\text { hs } \\
\text { (m) }\end{array}$ & Tipo de oleaje & $\begin{array}{c}\boldsymbol{H}_{s} \\
(\mathrm{~m})\end{array}$ & $\begin{array}{l}T_{p} \\
\text { (s) }\end{array}$ & $\begin{array}{l}\text { Rc } \\
\text { (m) }\end{array}$ & $\gamma_{\beta}$ & $\gamma_{s}$ \\
\hline 2 & 0.60 & Ondas enfocadas & 0.04 & 2.00 & 0.07 & 0.83 & 1 \\
\hline 3 & 0.60 & Ondas enfocadas & 0.04 & 2.00 & 0.17 & 0.83 & 1 \\
\hline
\end{tabular}

\section{Resultados}

Esta sección muestra la validación del modelo numérico con las mediciones de laboratorio y su aplicación para estudiar la sensibilidad de la estabilidad y funcionalidad de la estructura a su ubicación relativa al punto de enfocamiento.

\section{Validación del modelo numérico}




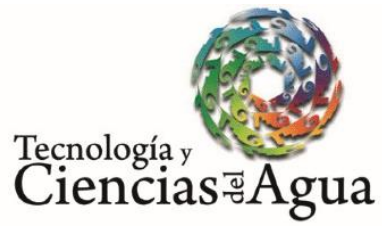

2021, Instituto Mexicano de Tecnología del Agua

Open Access bajo la licencia CC BY-NC-SA 4.0

(https://creativecommons.org/licenses/by-nc-sa/4.0/)

El modelo numérico fue comparado con las mediciones de superficie libre a lo largo del canal, mediciones de presiones alrededor de la estructura y rebase.

\section{Transformación del oleaje}

El oleaje generado corresponde al punto de enfocamiento teórico, de acuerdo con la Ecuación (5), localizado en $x=19 \mathrm{~m}$. Sin embargo, las mediciones en el canal de oleaje muestran que el enfocamiento sucede en $x=17 \mathrm{~m}$. En la Figura 8 se presenta la serie temporal de la superficie libre a diferentes posiciones a lo largo del canal correspondientes a los ensayos 1 ( $\sin$ estructura) y 2 (con estructura). Para el caso sin estructura, la transformación del tren de olas es reproducida de manera satisfactoria por el modelo numérico (Figuras $8 a-1$ y $8 b-1$ ), incluyendo la predicción del punto de enfocamiento (Figura 8c-1). La diferencia de $2 \mathrm{~m}$ de la ubicación del punto de enfocamiento entre el modelo lineal, los datos de laboratorio y el modelo numérico sugiere que las diferencias se encuentran principalmente asociadas con procesos de transformación del oleaje no-lineales. Baldock et al. (1996) reportaron un incremento en las diferencias entre el punto de enfocamiento teórico y el observado con el 
incremento de la no-linealidad del grupo de ondas, las cuales causan un efecto en la dispersión de las ondas.
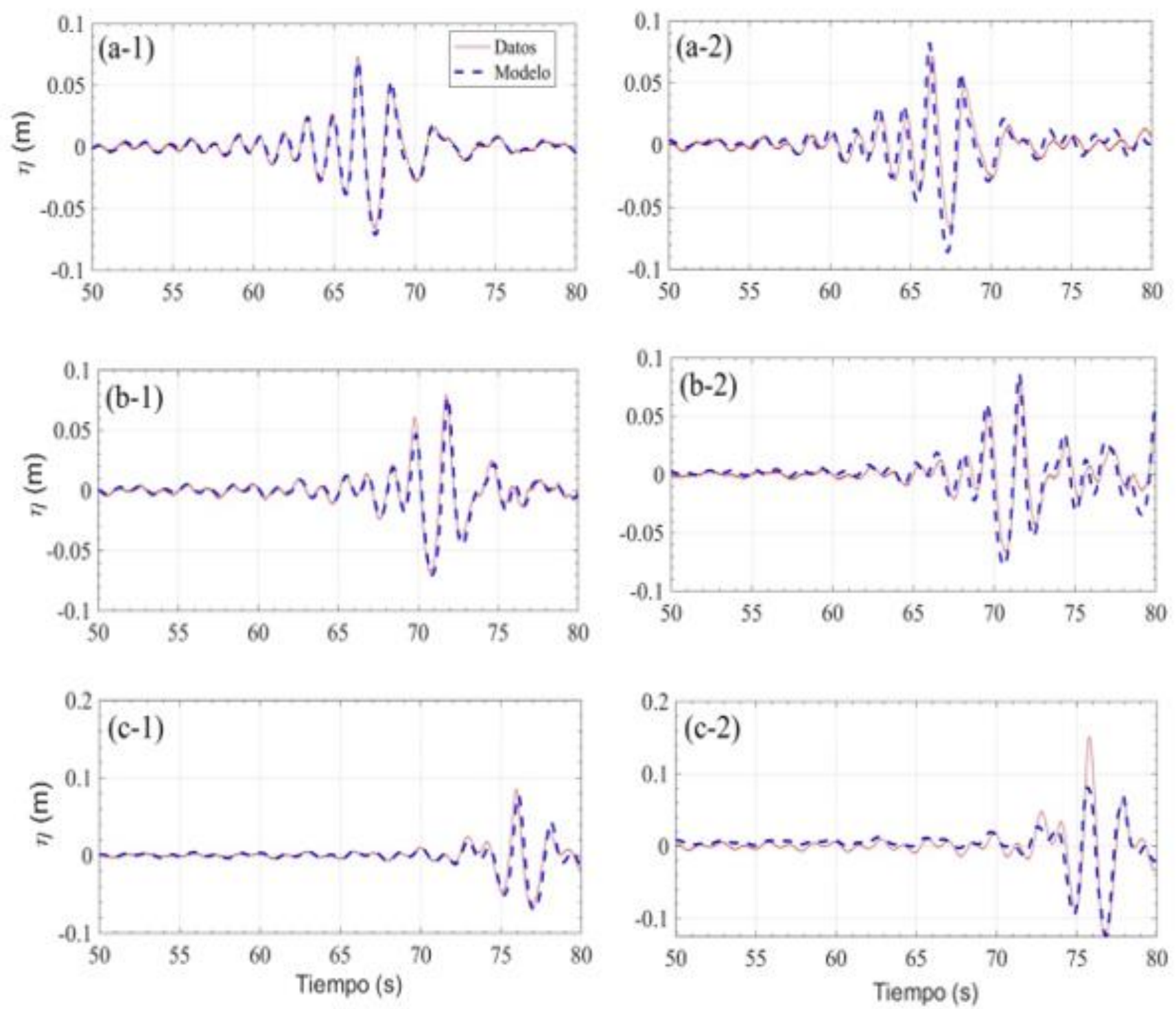

Figura 8. Comparación entre las mediciones y las predicciones del modelo numérico de la serie temporal de superficie libre en (a) $x=1 \mathrm{~m}$; (b) $8 \mathrm{~m}$, y (c) $17 \mathrm{~m}$ para la propagación de ondas enfocadas en el canal 


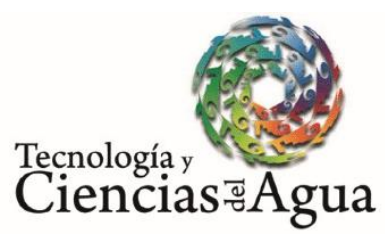

de oleaje para los casos en (1) ausencia y en (2) presencia de la estructura en $x=17 \mathrm{~m}$.

La Figura 8a-2-c-2 muestra las mediciones de superficie libre, realizados en presencia de la estructura (ensayo 2 ). El modelo numérico reproduce de manera satisfactoria los procesos asociados con la propagación del grupo de ondas (Figura $8 a-2$ y Figura $8 b-2$ ). El oleaje, al interactuar con la estructura, induce la rotura del oleaje, así como el rebase y la reflexión parcial. Es evidente el incremento de la altura de las olas en el ensayo 2 (Figura 8c-2), el cual está asociado con el proceso de reflexión del oleaje en la cara de la estructura. El modelo numérico predice de manera cualitativa la asimetría del oleaje y la transformación del grupo. Sin embargo, subestima la altura del oleaje en la cara de la estructura (ver Figura 8c-2).

\section{Campo de presiones}

Las presiones y subpresiones máximas alrededor de la estructura son necesarias para determinar su estabilidad. En la Figura 9 se muestra la comparación de las mediciones de presión en los 10 sensores instalados alrededor de la estructura durante el ensayo 2 contra las mediciones del 


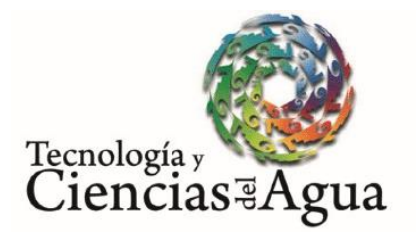

2021, Instituto Mexicano de Tecnología del Agua

Open Access bajo la licencia CC BY-NC-SA 4.0

(https://creativecommons.org/licenses/by-nc-sa/4.0/)

modelo numérico. Las mediciones muestran una atenuación de la presión dinámica a lo largo de la base de la estructura. El modelo numérico es capaz de reproducir la amplitud y la variación temporal de presiones tanto en los sensores que se encuentran sumergidos como en los que son inundados ocasionalmente debido a la interacción del oleaje con la estructura. El modelo reproduce la variación temporal de la presión en la cara de la estructura (Figura 9a-e). Las diferencias principales se presentan en el sensor P2 (Figura 9b), las cuales están asociadas con las limitaciones del modelo para predecir la superficie libre en esa posición (Figura 8c-2). 
Tecnología y

Ciencias $₫$ Agua
2021, Instituto Mexicano de Tecnología del Agua

Open Access bajo la licencia CC BY-NC-SA 4.0

(https://creativecommons.org/licenses/by-nc-sa/4.0/)
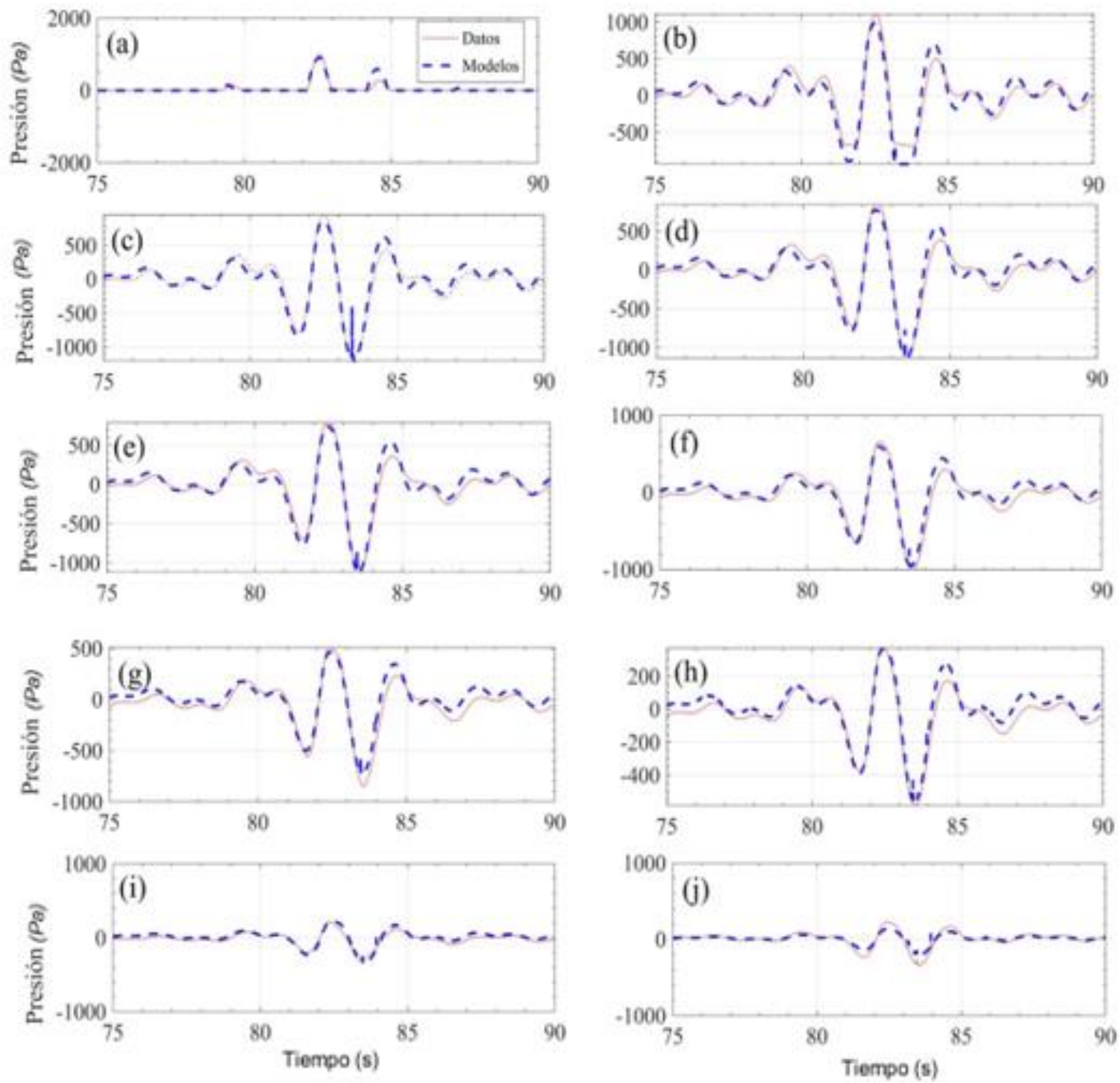

Figura 9. Comparación de la serie temporal de presiones entre las mediciones y el modelo numérico en los sensores ubicados en: la cara barlomar del dique (a: P1; b: P2; c: P3; d:P4; e: P5) y en la base del 
dique (f: P6; g: P7; h: P8; i: P9; j: P10). La ubicación de los sensores se detalla en la Figura 6.

La Figura 10 y la Tabla 5 muestran la comparación entre las presiones máximas medidas en el laboratorio y obtenidas por el modelo numérico. El modelo numérico subestima las presiones en los sensores P1 a P9.

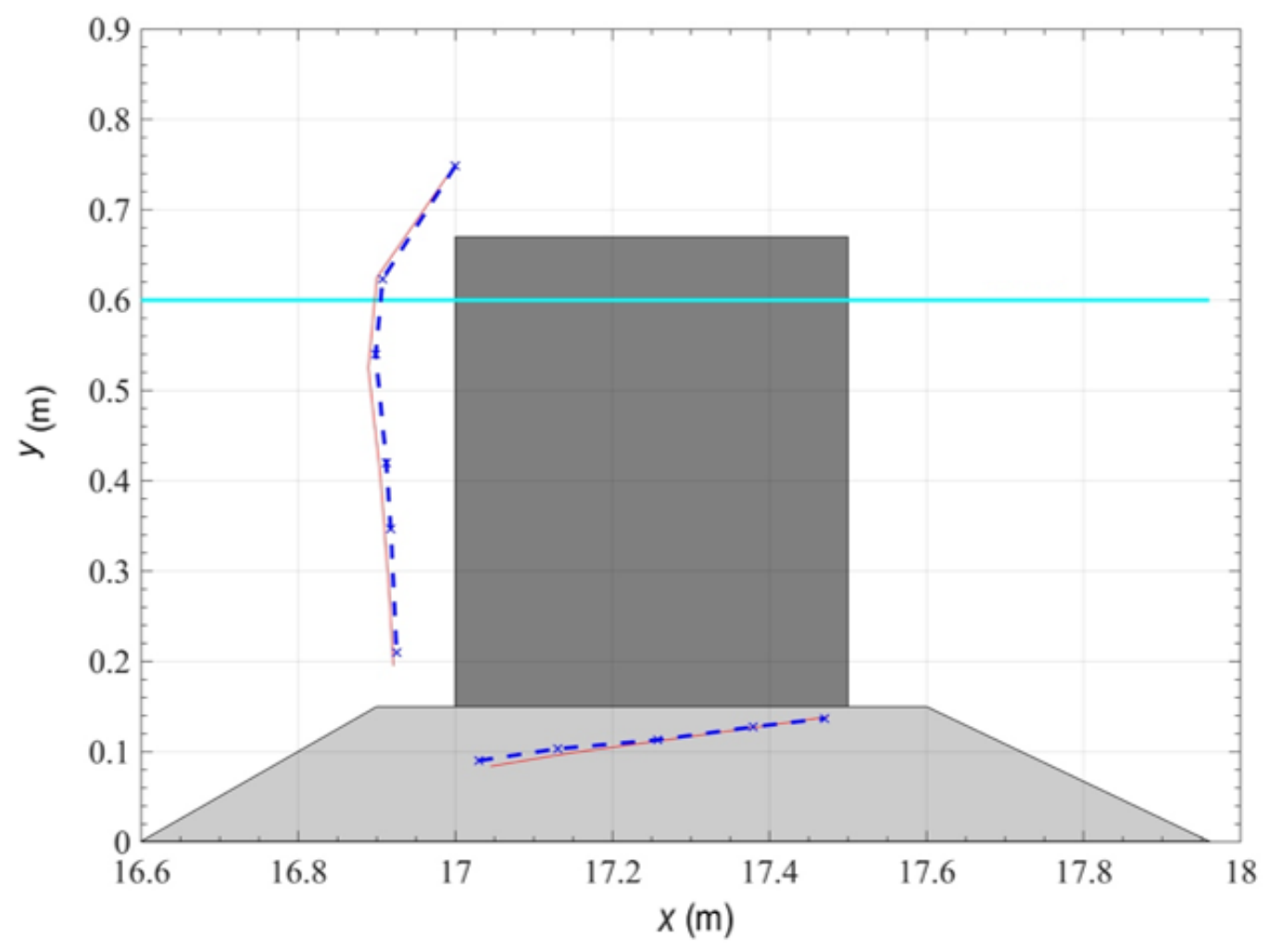

Figura 10. Presiones y subpresiones dinámicas máximas medidas en el ensayo 2 (línea continua) y modeladas (línea discontinua). Una distancia de $0.09 \mathrm{~m}$ equivale a $1 \mathrm{KPa}$. 
Tabla 5. Error absoluto y relativo de las presiones y subpresiones del modelo físico (Ensayo 2) y numérico (Caso2).

\begin{tabular}{|c|c|c|c|c|}
\hline Sensor & $\begin{array}{c}\text { Presiones } \\
\text { Modelo físico } \\
\text { (Pa) }\end{array}$ & $\begin{array}{l}\text { Presiones } \\
\text { Modelo } \\
\text { numérico } \\
(P a)\end{array}$ & $E_{a}=\left|P-P_{\text {Numérico }}\right|(P a)$ & $\begin{array}{c}E_{r}=\left|\frac{P-P_{\text {Numérico }}}{P}\right| \\
\times 100 \\
(\%)\end{array}$ \\
\hline P1 & 998.76 & 908.00 & 90.76 & 9.09 \\
\hline $\mathbf{P 2}$ & 1108.81 & 1014.00 & 94.81 & 8.55 \\
\hline P3 & 954.10 & 878.00 & 76.10 & 7.98 \\
\hline P4 & 849.10 & 788 & 61.10 & 7.20 \\
\hline P5 & 787.21 & 748.00 & 39.21 & 4.98 \\
\hline P6 & 660.03 & 597.00 & 63.03 & 9.55 \\
\hline P7 & 514.80 & 466.00 & 48.80 & 9.48 \\
\hline P8 & 373.00 & 366.00 & 7.00 & 1.88 \\
\hline P9 & 224.00 & 223.00 & 1.00 & 0.45 \\
\hline P10 & 111.02 & 131.00 & 19.98 & 18.00 \\
\hline & & Promedio & 50.18 & 7.71 \\
\hline
\end{tabular}

El modelo numérico presentó una subestimación en la superficie libre con respecto al modelo físico, que se refleja en la subestimación en las presiones y subpresiones máximas. Estos resultados son consistentes con el trabajo de Guanche (2007), quien reportó que la colocación de las piezas en la banqueta del dique puede influir en el resultado en caso de que la geometría difiera de la sección ideal simulada por el modelo. Aunado a ello, existen limitaciones para modelar la complejidad de la 


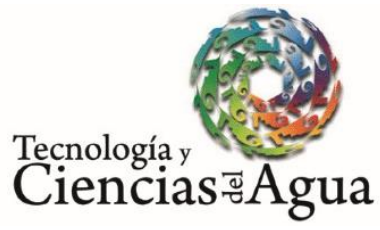

2021, Instituto Mexicano de Tecnología del Agua

Open Access bajo la licencia CC BY-NC-SA 4.0

(https://creativecommons.org/licenses/by-nc-sa/4.0/)

interacción de oleaje-estructura a través de parámetros del medio poroso influyen en los resultados del modelo numérico. Asimismo, los métodos de generación de oleaje en canales físicos y modelos numéricos pueden crear variaciones tanto en superficie libre, rebase y presiones, dependiendo de las limitaciones de los métodos debido a las características de cada uno. En los canales físicos puede ser una limitante la zona de generación, disipación, material de construcción del canal entre otros; y en los modelos numéricos, las condiciones que se consideran para generar la solución (Alves-Oliveira, 2012). Por lo tanto, existe una influencia en el método de generación de oleaje en los resultados obtenidos. Las diferencias entre el modelo numérico y físico se podrían resolver si se mejora el método de simulación, como se muestra en el trabajo de Lara, Ruju y Losada (2010), donde se modela el movimiento de la pala, acercándose más a la realidad.

\section{Rebase}

La funcionalidad de la estructura se evalúa a partir del rebase. El rebase se calculó con base en la medición del nivel realizada por el sensor S17 localizado en el vertedero para el ensayo 2. Para este caso, el rebase sucede de manera abrupta durante el enfocamiento del oleaje. La 


\section{Ciencias $₫$ Agua}

comparación del modelo numérico con las mediciones muestra una buena predicción, pero con una muy ligera subestimación (Figura 11 y Tabla 6).

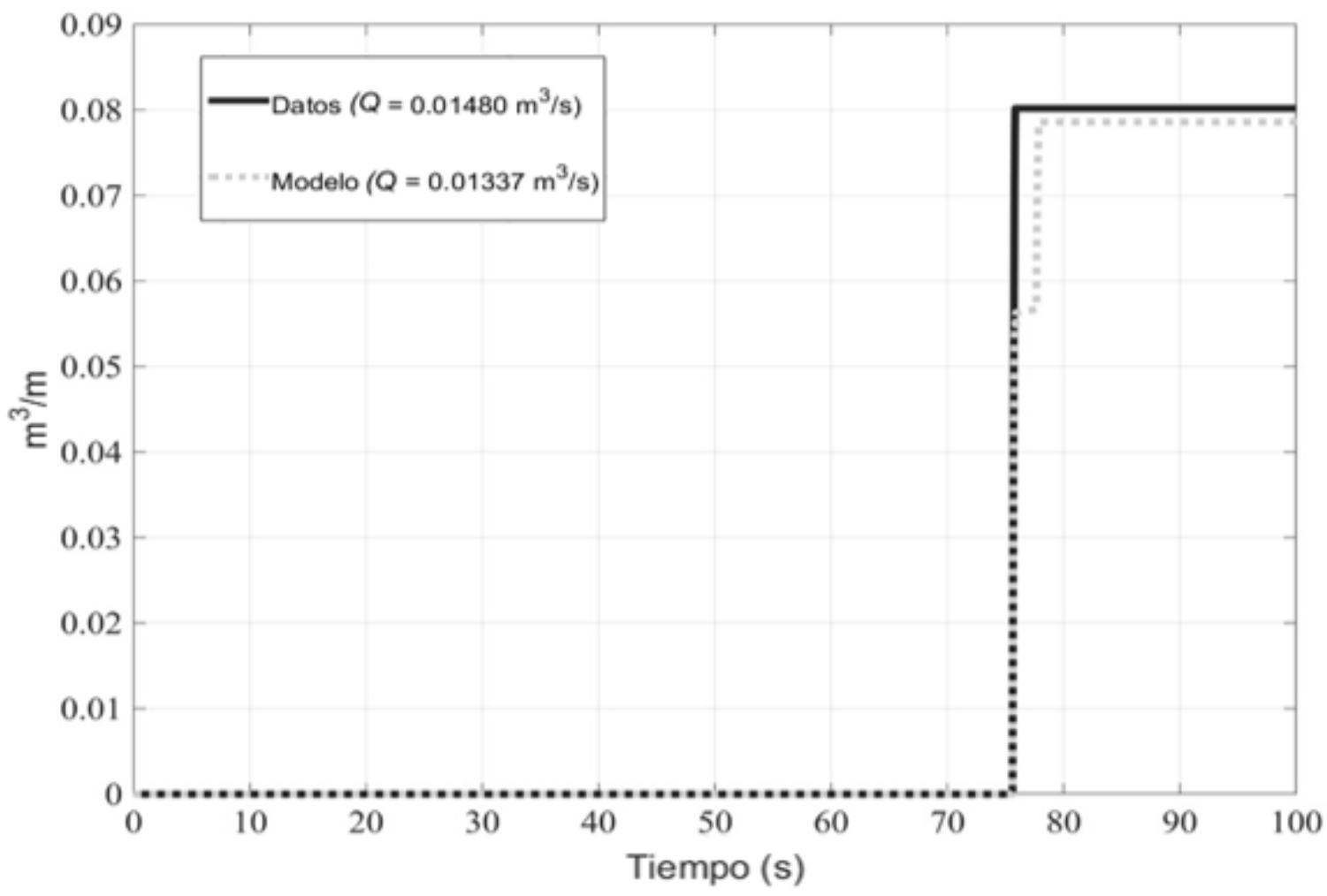

Figura 11. Comparación entre el modelo numérico y mediciones de laboratorio del rebase instantáneo.

Tabla 6. Caudales obtenidos del modelo físico (Ensayo 2) y numérico (Caso 2), que muestran su error absoluto y error relativo.

$\begin{array}{lllcl}\text { Ensayo Caso } & \begin{array}{c}\text { Tipo de } \\ \text { oleaje }\end{array} \quad \boldsymbol{Q} \text { físico } & \begin{array}{c}Q \\ \text { numérico }\end{array} & E_{a}=\left|Q-Q_{\text {Numéricol }}\right| & E_{r}=\left|\frac{Q-Q_{\text {Numéricoo }}}{Q}\right| \times 100 \\ (\%)\end{array}$




\begin{tabular}{|c|c|c|c|c|c|c|}
\hline 2 & 2 & $\begin{array}{c}\text { Ondas } \\
\text { enfocadas }\end{array}$ & 0.014 & 0.013 & 0.001 & 9.66 \\
\hline
\end{tabular}

\section{Aplicación del modelo numérico}

El modelo numérico reproduce de manera satisfactoria las mediciones de superficie libre, presiones, y rebase realizadas en el canal de oleaje (Figura 8, Figura 9, Figura 10 y Figura 11). Por lo tanto, en esta sección el modelo se empleó para simular otros casos que serían difíciles de implementar en el modelo físico. Se usó el mismo grupo de ondas enfocadas, pero se cambió la ubicación de la estructura con respecto al punto de enfocamiento teórico. En los casos modelados, la estructura se ubicó en las posiciones $x=7,14,20$ y 27 m (Tabla 2).

La Figura 12 muestra las presiones dinámicas y subpresiones dinámicas máximas para cada uno de los casos modelados. Se observa que para el mismo tren de oleaje, las presiones máximas tienen una variación de hasta el $14 \%$, dependiendo de la ubicación de la estructura $y$, por lo tanto, las condiciones del oleaje en rotura. Llama la atención el registro de la subpresión en el sensor P10 para el caso 3 (estructura localizada en $x=7 \mathrm{~m}$ ). Este valor se encuentra asociado con un pico en el modelo, posiblemente relacionado con un valor espurio. Al ignorar este pico, la tendencia es similar a los otros casos. Por otro lado, el rebase presenta diferencias aún más significantes entre casos, con diferencias de 
$45 \%$ (Figura 13). Con mayores alturas de oleaje incidente existe un incremento en las presiones y subpresiones máximas (Figura 12), y el rebase (Figura 13) en la estructura. Es importante resaltar que la presencia de la estructura afecta el punto de enfocamiento real debido al efecto de la reflexión y por lo tanto hace que la predicción sea muy complicada.
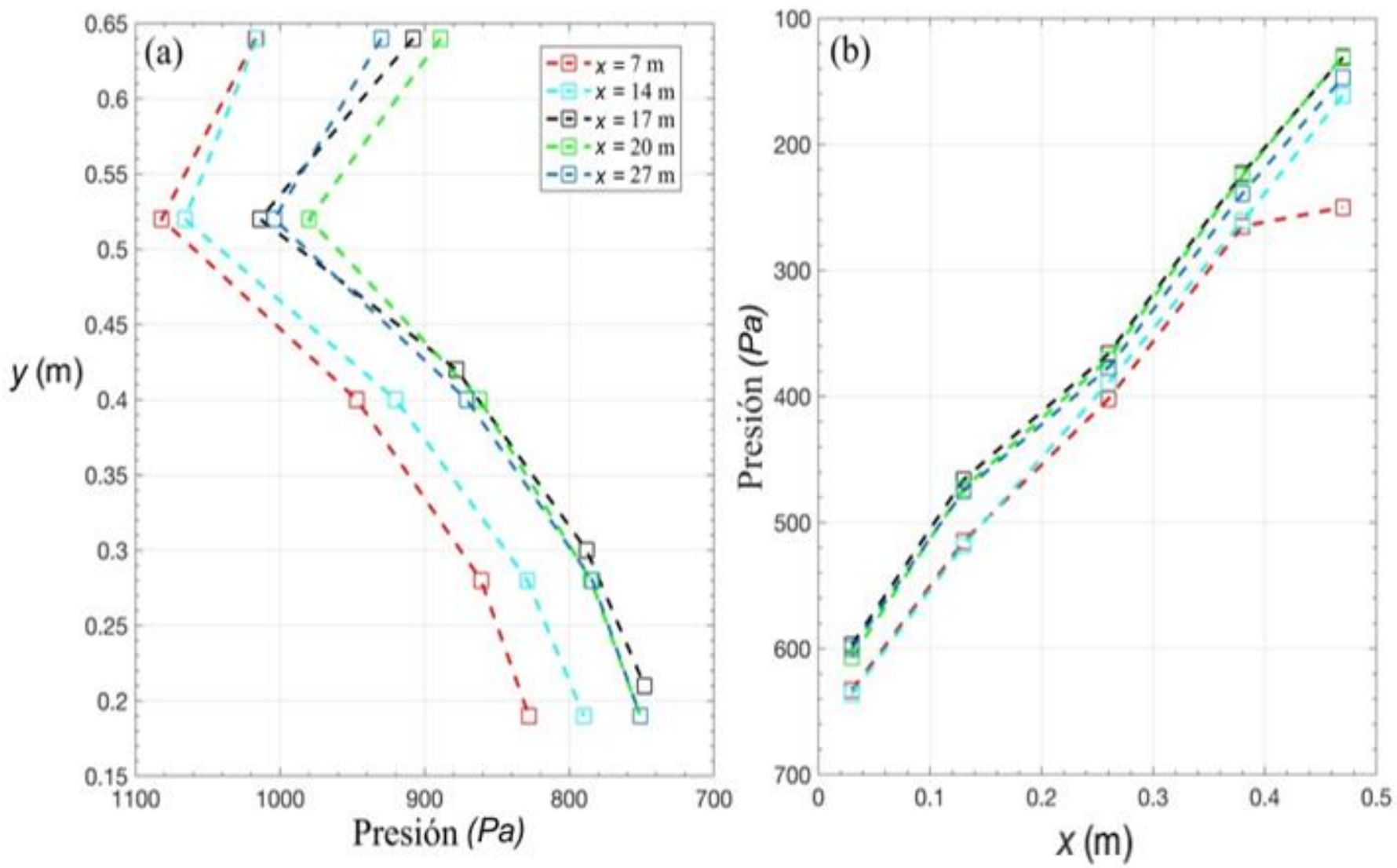

Figura 12. (a) Variación de la presión dinámica máxima en profundidad y (b) variaciones de las subpresiones dinámicas máxima con respecto a la distancia horizontal para los casos 2 al 6. 


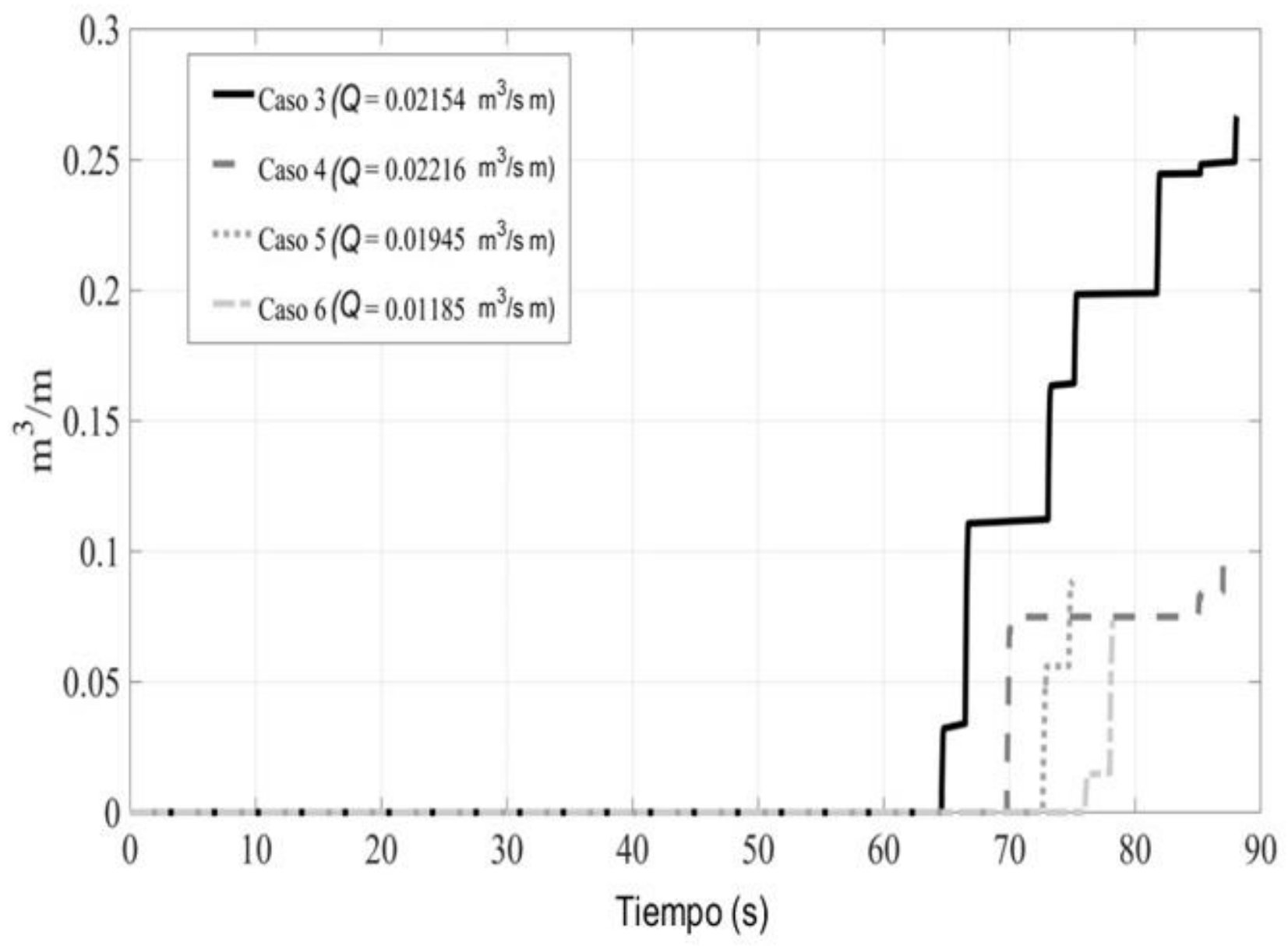

Figura 13. Evolución temporal del rebase instantáneo para: (a) caso 3, (b) caso 4, (c) caso 5 y (d) caso 6.

\section{Discusión}




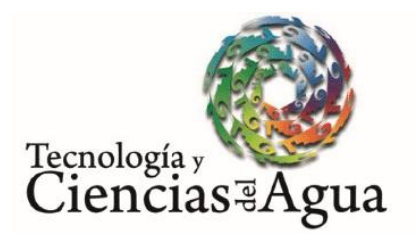

En esta sección se evalúa la capacidad de las formulaciones semianalíticas y empíricas utilizadas ampliamente para el diseño de estructuras. Para ello, se emplean las mediciones de presiones/subpresiones y rebase obtenidas en el canal de oleaje en los ensayos 2 y 3.

Las presiones dinámicas y subpresiones dinámicas máximas calculadas por el método de Goda (1974) subestiman de manera significativa las mediciones realizadas en el modelo físico durante dichos ensayos (Figura 14, Tabla 7 y Tabla 8). Asimismo, los rebases calculados por la formulación semiempírica de Franco y Franco (1999) muestran una subestimación del caudal en comparación con el modelo físico del ensayo 2 y 3 (Tabla 9). Las formulaciones semiempíricas no representan de manera correcta los resultados del laboratorio. Iribarren (2013) concluye que las formulaciones semiempíricas existentes, al ofrecer resultados con base en principios simplificados, pueden ser no capaces de dar respuesta a fenómenos complejos. 

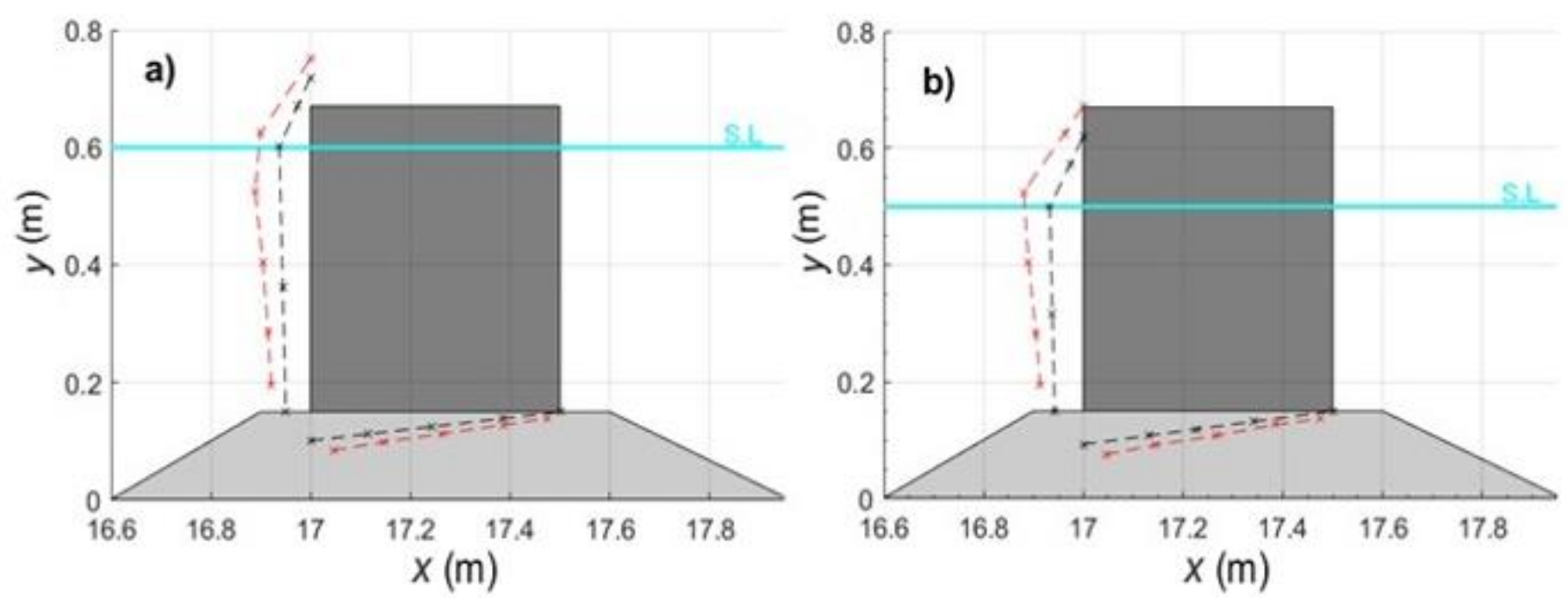

Figura 14. Comparación de las presiones del modelo analítico (Goda, 1974) con las presiones del laboratorio del (a) ensayo 2 y (b) ensayo 3, donde 0.09 m equivale a $1 \mathrm{KPa}$.

Tabla 7. Error absoluto y relativo de las presiones y subpresiones del modelo físico y Goda (1974) del ensayo 2.

\begin{tabular}{|c|c|ccc|}
\hline Sensor & $\begin{array}{c}\text { Presiones } \\
\text { Modelo físico } \\
(P a)\end{array}$ & $\begin{array}{c}\text { Modelo Goda } \\
(\mathbf{1 9 7 4 )}\end{array}$ & $\begin{array}{c}\boldsymbol{E}_{\boldsymbol{a}}=\left|\boldsymbol{P}-\boldsymbol{P}_{\text {Goda }}\right| \\
\mathbf{( P a )}\end{array}$ & $\boldsymbol{E}_{r}=\left|\frac{\boldsymbol{P}-\boldsymbol{P}_{\text {Goda }} \mid}{\boldsymbol{P}}\right| \times \mathbf{1 0 0}$ \\
\hline P1 & 998.76 & 0 & 998.76 & 100 \\
\hline P2 & 1108.81 & 625.80 & 483.01 & 43.56 \\
\hline P5 & 787.21 & 492.30 & 294.91 & 37.46 \\
\hline
\end{tabular}


Ciencias $₫$ Agua

\begin{tabular}{|c|c|c|c|c|}
\hline P6 & 660.03 & 491.70 & 168.33 & 25.50 \\
\hline P10 & 111.02 & 17.50 & 93.52 & 84.23 \\
\cline { 3 - 5 } & & Promedio & 407.70 & 58.15 \\
\hline
\end{tabular}

Tabla 8. Error absoluto y relativo de las presiones y subpresiones del modelo físico y Goda (1974) del ensayo 3.

\begin{tabular}{|c|c|c|c|c|}
\hline Sensor & $\begin{array}{c}\text { Presiones } \\
\text { Modelo físico } \\
\text { (Pa) }\end{array}$ & $\begin{array}{c}\text { Presiones } \\
\text { Modelo Goda } \\
(1974) \\
(\mathrm{Pa})\end{array}$ & $\begin{array}{c}E_{a}=\left|P-P_{\text {Goda }}\right| \\
\text { (Pa) }\end{array}$ & $\begin{array}{c}E_{r}=\left|\frac{P-P_{\text {Godada }}}{P}\right| \times 100 \\
(\%)\end{array}$ \\
\hline P1 & 378.16 & 0 & 378.16 & 100 \\
\hline $\mathbf{P 2}$ & 1195.99 & 673.2 & 522.79 & 43.71 \\
\hline P5 & 863.52 & 572.8 & 290.72 & 33.66 \\
\hline P6 & 747.80 & 571.9 & 175.90 & 23.52 \\
\hline P10 & 133.36 & 17. & 115.86 & 86.87 \\
\hline & & Promedio & 296.69 & 57.55 \\
\hline
\end{tabular}

Tabla 9. Caudales obtenidos del modelo Franco y Franco (1999), y del modelo físico de los ensayos 2 y 3 , mostrando su error absoluto y error relativo.

\begin{tabular}{|c|c|c|c|c|c|}
\hline Ensayo & $\begin{array}{c}\text { Tipo de } \\
\text { oleaje }\end{array}$ & QLab & QA & $\begin{array}{l}E_{a} \\
=\left|Q-Q_{\text {Franco }}\right|\end{array}$ & $\begin{array}{c}E_{r}=\left|\frac{Q-Q_{\text {Franco }}}{Q}\right| \times 100 \\
(\%)\end{array}$ \\
\hline 2 & $\begin{array}{c}\text { Ondas } \\
\text { enfocadas }\end{array}$ & 0.0148 & 0.000267 & 0.014533 & 98.19 \\
\hline
\end{tabular}




\begin{tabular}{|c|c|c|c|c|c|}
\hline 3 & $\begin{array}{c}\text { Ondas } \\
\text { enfocadas }\end{array}$ & 0 & 0.00000075 & 0.00000075 & 100 \\
\hline
\end{tabular}

\section{Conclusiones}

En el presente trabajo se realizó un estudio integral sobre la funcionalidad y estabilidad de una estructura costera al interactuar con un grupo de ondas enfocadas. Para ello, se llevaron a cabo ensayos en un canal de oleaje, se implementó un modelo numérico RANS, y se utilizaron formulaciones semiempíricas existentes en la literatura. A partir de los resultados se obtuvieron las siguientes conclusiones:

$>$ El punto de enfocamiento en aguas intermedias observado en el modelo físico difiere de la predicción basada en teoría lineal debido a la nolinealidad del oleaje.

$>$ El enfocamiento teórico es altamente sensible a la presencia de un dique vertical debido a la generación de una onda cuasi-estacionaria frente a ella.

> El modelo numérico RANS reproduce de manera satisfactoria la transformación no lineal de un grupo de ondas enfocadas en presencia de una estructura costera. En este trabajo, la presiones, subpresiones y 


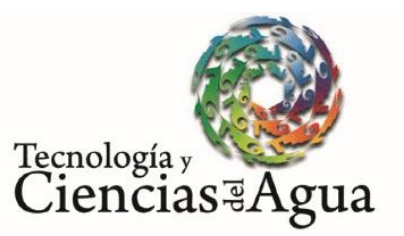

rebase fueron subestimados un $10 \%$. Sin embargo, el modelo presentó dificultades para la predicción de la superficie libre en el punto de enfocamiento para el caso con estructura.

$>$ El modelo numérico sugiere variaciones de hasta 14 y $45 \%$ de los valores máximos de presiones y caudales al variar la ubicación relativa de la estructura con relación al punto de enfocamiento teórico.

> Los modelos semiempíricos subestiman entre un 50 y $60 \%$ promedio las presiones $\mathrm{y}$ subpresiones $\mathrm{y} \approx 100 \%$ promedio en el rebase al ser comparado con observaciones para el caso de ondas enfocadas.

\section{Agradecimientos}

Agradecemos a Sergio Camilo Rendón Valdez por brindar apoyo técnico para realizar los ensayos en el canal de oleaje. B. Rodrigo Covarrubias Contreras agradece a Conacyt por la beca otorgada (No. 488695) para la realización de su posgrado en la UNAM. Alec Torres Freyermuth agradece a la DGAPA PAPIIT-UNAM por el financiamiento para realizar los ensayos en el canal de oleaje a través del proyecto IN101218. Gonzalo Uriel Martín Ruiz proporcionó soporte de TI. Finalmente, agradecemos a dos revisores anónimos que proporcionaron comentarios que contribuyeron a mejorar este artículo.

\section{Referencias}




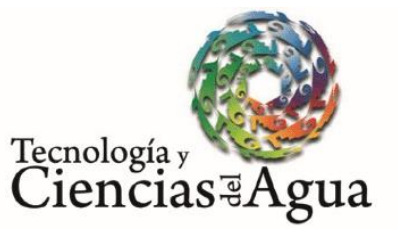

2021, Instituto Mexicano de Tecnología del Agua

Open Access bajo la licencia CC BY-NC-SA 4.0

(https://creativecommons.org/licenses/by-nc-sa/4.0/)

Alves-Oliveira, T. C. (2012). Generación de olas y estudio del rebase en un canal numérico de oleaje basado en el PFEM (tesis doctoral). Universitat Politécnica de Catalunya, Cataluña, España.

Amarachaharam, T. (2016). Numerical modelling of focussed wave hydrodynamics and focused wave-structure interaction with REEF3D (master's thesis). Norwegian University of Science and Technology, Trondheim, Norway.

Baldock, T. E. (2006). Long wave generation by the shoaling and breaking of transient wave groups on a beach. Proceedings of the Royal Society A: Mathematical, Physical and Engineering Sciences, 462, 1853-1876.

Baldock, T. E., Swan, C., \& Taylor, P. H. (1996). A laboratory study of non-linear waves on water. Philosophical Transactions of the Royal Society A: Mathematical, Physical and Engineering Sciences, 354, 649-676.

Chen, Q., Kirby, J. T., Dalrymple, R. A., Shi, F., \& Thornton, E. B. (2003). Boussinesq modeling of longshore currents, Journal of Geophysical Research, 108(C11), 3362, DOI: 10.1029/2002JC001308.

Franco, C., \& Franco, L. (1999). Overtopping formulas for caisson breakwaters with nonbreaking 3D waves. Journal of Waterway, Port, Coastal, and Ocean Engineering, American Society of Civil Engineers, 125(2), 98-108.

Goda, Y. (1974). New wave pressure formulae for composite breakwaters. Proceedings of the 14th International Coastal Engineering Conference, 3, 1702-1720. 


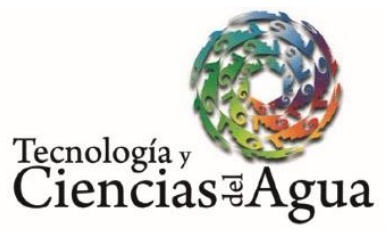

2021, Instituto Mexicano de Tecnología del Agua

Open Access bajo la licencia CC BY-NC-SA 4.0

(https://creativecommons.org/licenses/by-nc-sa/4.0/)

Goda, Y. (2004). Spread parameter of extreme wave height distribution for performance-based design of maritime structures. Journal of Waterway, Port, Coastal, and Ocean Engineering, American Society of Civil Engineers, 130(1), 29-38.

Gornitz, V. (1991). Global coastal hazards from future sea level rise. Palaeogrography, Palaeoclimatology, Palaeoecology, 89(4), 379398.

Guanche, R. (2007). Análisis de la funcionalidad y estabilidad de obras marítimas mediante un modelo numérico basado en las ecuaciones de Reynolds (tesis doctoral). Universidad de Cantabria, Cantabria, España.

Guanche, R., Losada, I., \& Lara, J. (2009). Numerical analysis of wave loads for coastal structure stability. Coastal Engineering, 56(5-6), 543-558. DOI: 10.1016/j.coastaleng.2008.11.003

Higuera, P., Lara, J., \& Losada, I. (2014). Three-dimensional interaction of waves and porous coastal structures using OpenFOAM $®$. Part II: Application. Coastal Engineering, (83), 259-270.

Hsu, T.-J., Sakakiyama, T., \& Liu, P.-F. (2002). A numerical model for wave motions and turbulence flows in front of a composite breakwater. Coastal Engineering, 46, 25-50.

Iribarren, E. (2013). Elementos para una nueva metodología de cálculo de diques verticales (tesis doctoral). Universidad Politécnica de Madrid, Madrid, España.

Jiménez, C. (2010). Criterios de diseño de rompeolas de berma, bajo el concepto de oleaje irregular (tesis de maestría). Instituto Politécnico Nacional, México, D.F., México. 


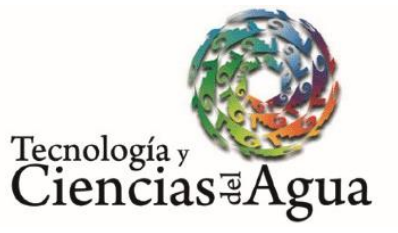

Kobayashi, N., \& Wurjanto, A. (1992). Irregular wave set up and run-up on beaches. Journal of Waterway, Port, Coastal, and Ocean Engineering, 118, 368- 386.

Lara, J. L., García, N., \& Losada, I. J. (2006). RANS modelling applied to random wave interaction with submerged permeable structures. Coastal Engineering, 53(5-6), 395-417.

Lara, J. L., Ruju, A., \& Losada, I. J. (2010) Reynolds averaged NavierStokes modelling of long waves induced by a transient wave group on a beach. Proceedings of the Royal Society A: Mathematical, Physical and Engineering Sciences, (2011), 467, 1215-1242. Recuperado de https://royalsocietypublishing.org/doi/pdf/10.1098/rspa.2010.033 1

Li, M., Zhao, X., Ye, Z., Lin, W., \& Chen, Y. (2018). Generation of regular and focused waves by using an internal wave maker in a CIP-based model. Ocean Engineering, 167, 334-347. Recuperado de www.elsevier.com/locate/oceaneng

Lin, P., \& Liu, P. L.-F. (1998). A numerical study of breaking waves in the surf zone. Journal of Fluid Mechanics, (359), 239-264.

Longuet-Higgins, M. (1974). Breaking waves - in deep or shallow water. Proceedings of the Tenth Naval Hydrodynamics Symposium, Office of Naval Research, 597-605.

Losada, I., Lara, J., Guanche, R., \& González-Ondina, J. (2008). Numerical analysis of wave overtopping of high mound breakwaters. Coastal Engineering, (55), 47-62. 


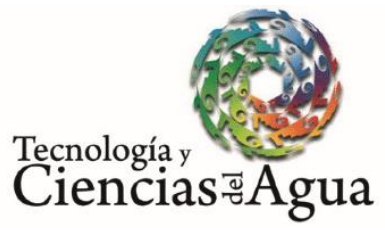

Nikolkina, I., \& Didenkulova, I. (2011). Rogue waves in 2006-2010. Natural Hazards and Earth System Sciences, (11), 2913-2924.

Palemón-Arcos, L., Torres-Freyermuth, A., Chang, K.-A., PastranaMaldonado, D., \& Salles, P. (2014). Modeling wave-structure interaction and its implication in offshore structure stability. Proceedings of the Twentey-Foruth International Offshore and Polar Engineering Conference, 1419-1424.

Palemón-Arcos, L., Torres-Freyermuth, A., Pedrozo-Acuña, A., \& Salles, P. (2015). On the role of uncertainty for the study of wave-structure interaction. Coastal Engineering, 106, 32-41. Recuperado de www.elsevier.com/locate/coastaleng

Rapp, R., \& Melville, W. (1990). Laboratory measurements of deep-water breaking waves. Philosophical Transactions of the Royal Society of London. Series A, Mathematical and Physical Sciences, 331, 735800. Recuperado de royalsocietypublishing.org

Ryu, Y., \& Chang, K. (2008). Green water void fraction due to breaking wave impinging and overtopping. Experiments in Fluids, 45(5), 883898.

Stagonas, D., Buldakov, E., \& Simons, R. (2018). Experimental generation of focusing wave groups on following and adversesheared currents in a wave-current flume. Journal of Hydraulic Engineering, 144(5), 1-11.

Torres-Freyermuth, A., Losada, I. J., \& Lara, J. L. (2007). Modeling of surf zone processes on a natural beach using Reynolds-Averaged Navier-Stokes equations. Journal of Geophysical Research, 112, C09014. 


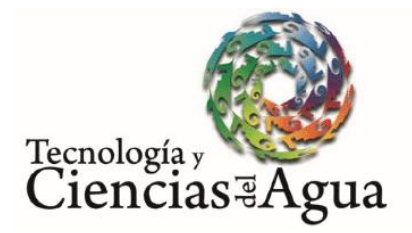

2021, Instituto Mexicano de Tecnología del Agua

Open Access bajo la licencia CC BY-NC-SA 4.0

(https://creativecommons.org/licenses/by-nc-sa/4.0/)

Van-den-Boomgaard, M. (2003). Wave focussing in a laboratory flume (master's thesis). Technological University of Delft, Delft, The Netherlands.

Whittaker, C., Fitzgerald, C., Raby, A., Taylor, P., \& Borthwick, A. (2018). Extreme coastal responses using focused wave groups: Overtopping and horizontal forces exerted on an inclined seawall. Coastal Engineering, 140, 292-305. Recuperado de www.elsevier.com/locate/coastaleng

Whittaker, C., Fitzgerald, C., Raby, A., Taylor, P., Orszaghova, J., \& Borthwick, A. (2017). Optimisation of focused wave group runup on a plane beach. Coastal Engineering, 121, 44-55. Recuperado de www.elsevier.com/locate/coastaleng 